\title{
New Developments in the Quantization of Supersymmetric Solitons (Kinks, Vortices and Monopoles)
}

\author{
Anton Rebhan, \\ Institut für Theoretische Physik, Technische Universität Wien, \\ Wiedner Haupstr. 8-10, A-1040 Vienna, Austria \\ Peter van Nieuwenhuizen, \\ C.N.Yang Institute for Theoretical Physics, State University of New York, \\ Stony Brook, NY 11794-3840, USA \\ and Robert Wimmer \\ Institut für Theoretische Physik, Universität Hannover, \\ Appelstr. 2, D-30167 Hannover, Germany
}

Received on 2 March, 2004

\begin{abstract}
We discuss the one-loop quantum corrections to the mass $M$ and central charge $Z$ of supersymmetric (susy) solitons: the kink, the vortex and the monopole. Contrary to previous expectations and published results, in each of these cases there are nonvanishing quantum corrections to the mass. For the $N=1$ kink and the $N=2$ monopole a new anomaly in $Z$ rescues BPS saturation $(M=Z)$; for the $N=2$ vortex, BPS saturation is rescued for two reasons: (i) the quantum fluctuations of the Higgs field acquire a nontrivial phase due to the winding of the classical solution, and (ii) a fermionic zero mode used in the literature is shown not to be normalizable.
\end{abstract}

\section{An introduction to new develop- ments in quantum field theory for susy solitons}

Solitons [1] have recently come back in the center of attention of quantum field theory (QFT) because in a certain class of supersymmetric (susy) field theories (the ones with $N=2$ susy) dualities between field theories with pointparticles and field theories with solitons allowed calculation of some nonperturbative effects [2]. Let us begin by defining what we mean by a soliton:

Definition 1: a soliton is a nonsingular timeindependent solution of the classical field equations in Minkowski spacetime with finite energy.

Solitons can be viewed as extended particles ("lumps") which should clearly have finite mass (= finite energy at rest). We shall only consider relativistic field theories. There exist also time-dependent solutions with finite energy (the "breather" solution in the sine-Gordon model, for example), but we consider only time-independent solitons. One can, of course, boost solitons in a relativistic theory, and obtain then moving solitons, but since one can always choose a Lorentz frame in which they are at rest, we restrict our attention to only time-independent solutions.
A soliton is closely related to an instanton. The later is defined as follows:

Definition 2: an instanton is a nonsingular solution of the classical field equations in Euclidean space with finite action.

Because instantons have finite action, they contribute already at the classical level to the path integral. This is the reason for the requirement of finite action. It is clear that a soliton in $n+1$ dimensions is an instanton in $n$ dimensions: since the time coordinate plays no role in the soliton solutions, the space integral in Minkowski space can also be viewed as an integral over Euclidean space, and the energy $E=\int d^{n} x \mathcal{H}(x)=\int d^{n} x(p \dot{q}-\mathcal{L})$ is equal to the action $S=-\int d^{n} x \mathcal{L}$.

We shall discuss three solitons:

1) the kink in $1+1$ dimensions with $N=1$ susy

2) the vortex in $2+1$ dimensions with $N=2$ susy

3) the monopole in $3+1$ dimensions with $N=2$ susy

There exist also susy extensions of these solitons with more susy ( $N=2$ for the kink, $N=4$ for the monopole) or less susy ( $N=1$ for the vortex). In addition, discussions have 
been given about the quantum corrections to these solitons without fermions and without susy.

All three models have recently led to surprising new insights in the quantization of solitons. In particular, a new anomaly was discovered in the quantum corrections to the central charge of the susy algebras for these models. The solitons have not only a mass $M$ but also a central charge $Z$ [3]. The quantum mass of a soliton is obtained by evaluating the vacuum expectation value of the Hamiltonian. The latter depends on the soliton background because one decomposes quantum fields $\phi$ into a background field and a quantum field. The quantum fluctuations appear quadratically (and of course also at higher orders) in the action, and thus the quantum correction to the mass is to first order in $\hbar$ the sum over the zero point energies of all the fluctuations. The quantum central charge arises as follows. The susy generators are the Noether charges for rigid susy, and as any Noether charge they are the space integrals over the time components of the Noether currents. They are expressed in terms of the Heisenberg fields $\phi$. Using equal-time canonical (anti) commutation relations, one finds then for example for the kink at the full quantum level before regularization

$$
\begin{aligned}
& \left\{Q^{ \pm}, j^{ \pm}(x)\right\}=\mathcal{H}(x) \pm \zeta(x) \\
& j^{ \pm}=\dot{\phi} \psi_{ \pm}+\left(\partial_{x} \phi \pm U\right) \psi_{ \pm} \\
& Q_{ \pm}=\int j_{ \pm}(x) d x \\
& \zeta(x)=\partial_{x} \phi(x) U(x)=\partial_{\sigma} W(\phi)
\end{aligned}
$$

where $U$ is a potential quadratic in $\phi$ and $W(\phi)=$ $\int U(\phi) d \phi$.

Classically, $M=Z$, the well-known BPS bound, but quantum mechanically both $M$ and $Z$ get quantum corrections. At first sight (or thought) it is surprising that $Z$ gets any quantum corrections at all, because classically $Z$ is the space integral of a total space-derivative. So it gets its contributions from far away from the soliton, and if one can neglect the presence of the soliton, how can one still get nonvanishing corrections? The answer (one answer) is that the central charge is a composite operator, and applying point splitting as a regularization scheme, the total derivative ceases to be a total derivative, and one gets then quantum corrections also from the region where the soliton is.

We shall not use point splitting as regularization scheme, but dimensional regularization. There are actually two versions of dimensional regularization

(1) ordinary ('t Hooft-Veltman-Bollini-Giambiagi) dimensional regularization $[4,5]$ where $n$ becomes larger than $n_{0}=2,3$ or 4 for our models

(2) dimensional reduction [6], where one lets $n$ get smaller than $n_{0}$. Vector fields $A_{\mu}$ decompose then into $n$-dimensional vector fields with $0 \leq \mu \leq n$ and scalar fields (called $\epsilon$-scalars) where $n \leq \mu \leq n_{0}$ (and $\left.n_{0}-n=\epsilon\right)$.

If $n>n_{0}$ one needs a model which remains susy in higher dimensions if we want to preserve susy at the regularized level. This is in general not possible because the number of components of spinors grows much faster in higher dimensions than that of bosons. However, in the low dimensions we consider there are possibilities. For example, the $N=1$ (more precisely $N=(1,1)$ ) susy kink in $1+1$ dimensions remains $N=1$ susy in $2+1$ dimensions because spinors have 2 components both in 2 and in 3 dimensions. For the vortex we preserve $N=1$ susy in $3+1$ dimensions by starting with an $N=2$ model in $2+1$ dimensions $(2 \times 2=1 \times 4)$. Finally, for the monopole we get a minimal susy model in 5+1 dimensions (with complex unconstrained 4-component chiral spinors) by starting with an $N=2$ model in $3+1$ dimensions with two real 4-component spinors (or, equivalently, two complex 2-component spinors).

If $n<n_{0}$, the models remain automatically susy because one does not change the number of bosonic and fermionic field components (one only reinterprets some components of vector fields as $\epsilon$-scalars as we have discussed). So much for how we deal with susy in the two versions of dimensional regularization. We wish to preserve (ordinary, see below) susy, and the best way to preserve it is to preserve it manifestly, by using models which remain susy even after regularization.

How can one understand that an anomaly in $Z$ is present? Anomalies form multiplets in susy, and these anomaly multiplets contain the trace anomaly and the conformal susy anomaly $\gamma \cdot j$. Here a small explanation is needed. Supersymmetry is the same as translational symmetry into the fermionic directions in superspace, and like ordinary translational symmetry, there is no anomaly in ordinary susy: $\partial^{\mu} j_{\mu}$ (susy) $=0$ at the quantum level. However, in massless theories one also has conformal susy (the fermionic counterpart of scale invariance and conformal boost invariance), and the conformal susy current is $j_{\mu}($ conf $)=\left(x^{\rho} \gamma_{\rho}\right) j_{\mu}$ (susy). Clearly, $\partial^{\mu} j_{\mu}$ (conf) $=\gamma^{\mu} j_{\mu}$ (susy) if ordinary susy is free from anomalies. In massless (conformal) theories the ordinary susy current satisfies at the classical level the relation $\gamma^{\mu} j_{\mu}$ (susy) $=0$. For example, in the $\mathrm{WZ}$ model in $1+1 \mathrm{di}-$ mensions, $j_{\mu}$ (susy) $=(\not \partial \varphi) \gamma_{\mu} \psi$, and since $\gamma^{\mu} \gamma_{\rho} \gamma_{\mu}=0$ in $1+1$ dimensions, one has $\gamma \cdot j$ (susy) $=0$. In $3+1$ dimensions the WZ model yields $j_{\mu}$ (susy) $=F_{\rho \sigma} \gamma^{\rho \sigma} \gamma_{\mu} \psi$, and since $\gamma^{\mu} \gamma^{\rho \sigma} \gamma_{\mu}=0$ in $3+1$ dimensions, also here $\gamma \cdot j$ (susy) $=0$. At the quantum level, there can be anomalies in $j_{\mu}$ (susy) (the current $j_{\mu}$ (conf) is no longer conserved, equivalently $\gamma^{\mu} j_{\mu}$ (susy) is no longer vanishing).

What is the technical reason that there is an anomaly, how does it appear when one calculates? Let us recall that an anomaly usually appears as 0/0: 0 because classically there is no violation, and 1/0 because a quantum field has infinitely many degrees of freedom (there are no anomalies in quantum mechanics, see however [70]). Consider now first ordinary dimensional regularization. It is known that translations in higher dimensions become central charges in lower dimensions. For example, the susy kink model has 3 translations in 3 dimensions, which become 2 translations and one central charge if one performs dimensional reduction (setting the coordinate $x^{3}$ to zero). This seems to doom the prospects of anomalies in $Z$, because we already mentioned that there are no anomalies in the translational symmetry. However, the presence of the soliton in the background 
polarizes the fermionic excitations in the extra dimensions $[7,8]$. This phenomenon has the same origin as the quantum Hall effect, and as a result the left-moving and the rightmoving modes of the fermions have different normalizations on the domain wall created by the soliton. One word about domain walls $[5,9,10,11,12]$ : if one moves from $1+1$ to $2+1$ dimensions, with coordinates $(x, y, t)$ and $y$ is the new coordinate, then the soliton $\varphi(x)$ remains a solution with finite energy per unit of length in the extra dimension. In $1+1$ dimensions the energy density of the soliton solution $\varphi(x)$ is located around $x=0$, so in $2+1$ dimensions it is located around the "wall" (line in this case) $x=0$ but any $y$. One must then solve the Dirac equation in $2+1$ dimensions, and in this way one discovers the polarization. (In string theory, Horava and Witten [13], and others have used this effect for other purposes ${ }^{1}$ ). A detailed calculation reveals that for the kink and the monopole there is indeed an anomaly coming from the polarization of domain wall fermions. Two remarks should quickly be made: (1) in odd dimensions there are no anomalies, so how $Z$ gets a contribution for the vortex should at this point not yet be clear to the uninformed reader, and furthermore, (2) there are also massless fermions on the domain wall; they are chiral (they only move in one direction) but these fermions do not contribute to the anomaly because in dimensional regularization massless tadpole integrals vanish. Only the polarization of the massive domain wall fermions yields an anomaly.

In the calculation of quantum corrections to $M$ and $Z$ one should let $n$ tend to $n_{0}$, so $\epsilon=n_{0}-n$ tends to zero. However the sum of all polarizations is divergent. As the reader may be now anticipate one indeed finds a total correction of the form $\epsilon / \epsilon$ which is finite. Many other regularization schemes have been analyzed, and also in these schemes one finds an anomaly in $Z$ and the value of this anomaly is the same in all cases.

The picture becomes now clear in even dimensions (kink, monopole). There are nonvanishing quantum corrections to the mass, partly due to nonanomalous corrections (which however vanish for kink and monopole in the most widely used renormalization scheme) and partly due to the trace anomaly. There are also nonvanishing corrections to the central charge, due to the central charge anomaly (which sits in the same multiplet as the conformal susy anomaly and the trace anomaly). Both corrections are equal: the classical BPS bound also holds at the quantum level.

In odd dimensions, in particular for the vortex, there is another reason why there is a nonvanishing correction to $Z$. There cannot be an anomaly, as we already mentioned, but now the soliton deforms the quantum fluctuations of the scalar (Higgs) field such that the latter acquires an extra phase. This phase has a nontrivial space dependence (a dependence on the angles which can not be removed continuously by a gauge transformation which is everywhere regular). This twist remains far away from the vortex and does give a nonvanishing contribution to the integral of the total derivative. The result is $M=Z$ also at the one loop level.
One would like to know whether there is an explanation for this equality. This involves fermionic zero modes, let us first define them.

\section{Definition 3: A zero mode is a time- independent solution of the linearized field equation for the quantum fluctuations which is normalizable and nonsingular.}

Bosonic zero modes can be obtained by making a symmetry transformation on the soliton, for example shifting or rotating the classical solution. Many, but not all, fermionic zero modes can be obtained by making an ordinary susy transformation of the soliton. (For single-instantons there exist fermionic zero modes for $S U(n)$ with $n \geq 3$ which do not come from susy).

Arguments have been given in the literature that the equality of $M$ and $Z$ for the susy vortex at the one-loop level is a mystery because one can count how many fermionic zero modes there are in this model and it has been claimed that there are two, rather than (as expected) one. With two fermionic zero modes the equality $M=Z$ could indeed not be explained; it could be due to some as yet not known symmetry, or it might just be an accident. However, we have shown that the second fermionic zero mode is not normalizable at the origin. Hence there is only one fermionic zero mode, and this means that the equality $M=Z$ which we obtained by detailed calculations using quantum field theory for extended objects, is, in fact, a direct consequence of ordinary supersymmetry at the quantum level.

So far we discussed ordinary dimensional regularization with $n \geq n_{0}$. We already discussed that dimensional reduction with $n \leq n_{0}$ preserves susy. The number of field components of fermions and bosons remains fixed (and one should treat some vector bosons as $\epsilon$ scalars as we discussed). The identity $\gamma^{\mu} \gamma_{\rho} \gamma_{\mu}=0$ remains valid because the index $\mu$ keeps running from 0 to 2 , instead of from 0 to $n_{0}$. (If this index would have been due to a derivative such as $j_{\mu}=\partial_{\mu} \varphi \psi$ then $\mu$ should only run up to $n$, but $j_{\mu}=\not \partial \varphi \gamma_{\mu} \psi$ and so $\mu$ must run up to $n_{0}$ ). This raised a problem many decades ago: it seemed that there was no conformal susy anomaly in dimensional reduction. Some people proposed wild solutions: breakdown of cyclicity of the trace operation or other drastic measures. Actually, the solution is conventional, although subtle: there are evanescent counter terms [14] for the currents. These are counter terms such as $\Delta j_{\mu}=\frac{1}{\epsilon} \not \partial \varphi \gamma_{\hat{\mu}} \psi$ where the index $\hat{\mu}$ only runs over $\epsilon$ values (namely from $n$ to $n_{0}$ ). One cannot write $\gamma_{\hat{\mu}}$ itself as $\epsilon$ times a finite quantity, but inside loop graphs the effect of $\gamma_{\hat{\mu}}$ is to supply a factor $\epsilon$. Thus $\Delta j_{\mu}$ yields finite contributions. If one requires that ordinary susy is preserved, one must satisfy $\partial^{\mu} j_{\mu}$ (susy) $=0$ in dimensional reduction, and then one must renormalize the susy current by adding an evanescent counter term. This counter term, and not the original loop graph, yields the anomaly. In ordinary dimensional regularization the situation is just the reverse: there the loop graph yields the anomaly (as discussed in text books) and

\footnotetext{
${ }^{1}$ They considered 2 branes in $d=11$ supergravity with a chiral spinor on one brane and an antichiral spinor on the other (this combination cannot be avoided because there are no chiral spinors in odd dimensions). Then they sent one brane to infinity, applied dimensional reduction, and found in this way chiral spinors in 4 dimensions.
} 
the counter terms do not contribute to the anomaly. One can construct the whole anomaly multiplet, and one finds then that the evanescent counter term $\Delta j_{\mu}$ in the conformal susy current yields a finite nonsingular contribution to the central charge anomaly. This finite term is the anomaly in $Z$, and the value of this anomaly is the same as that obtained from ordinary dimensional regularization. To avoid misunderstanding: we also directly computed this anomaly using dimensional reduction, but as the preceding discussion shows, one can also obtain it by making susy transformations of the anomaly $\gamma \cdot j$ (susy).

We have written several papers on these subjects, and also published some reviews $[15,16]$. For a gentle introduction we recommend [15]. In the remaining sections we focus on the kink, vortex, and monopole, respectively, using susy-preserving dimensional regularization methods.

\section{The (susy) kink.}

The calculation of quantum corrections to the mass of a supersymmetric (susy) kink and to its central charge has proved to be a surprisingly subtle problem, and it took protracted struggles to fully understand it in the various methods that had been employed.

Initially it was thought that supersymmetry would lead to a complete cancellation of quantum corrections [17] and thereby guarantee Bogomolnyi-Prasad-Sommerfield (BPS) saturation at the quantum level. Then, by considering a kinkantikink system in a finite box and regularizing the ultraviolet divergences by a cutoff in the number of the discretized modes, Schonfeld [18] found that there is a nonzero, negative quantum correction at one-loop level, $\Delta M^{(1)}=$ $-m /(2 \pi)$. Most of the subsequent literature [19] considered instead a single kink directly, using (usually implicitly) an energy-momentum cutoff which gave again a null result. A direct calculation of the central charge [20] also gave a null result, apparently confirming a conjecture of Witten and Olive [3] that BPS saturation in the minimally susy $1+1$ dimensional case would hold although arguments on multiplet shortening naively do not seem to apply.

In Ref. [21] two of the present authors noticed a surprising dependence on the regularization method, even after the renormalization conditions have been fully fixed. In particular it was found that the naive energy-momentum cutoff as used in the susy case spoils the integrability of the bosonic sine-Gordon model [22]. Using a mode regularization scheme and periodic boundary conditions in a finite box instead led to a susy kink mass correction $\Delta M^{(1)}=$ $+m(1 / 4-1 / 2 \pi)>0$ (obtained previously also in Ref. [23]) which together with the null result for the central charge appeared to be consistent with the BPS bound, but implying nonsaturation. Subsequently it was found by two of us together with Nastase and Stephanov [24] that the traditionally used periodic boundary conditions are questionable. Using instead topological boundary conditions which are invisible in the topological and in the trivial sector together with a "derivative regularization"2 indeed led to a different result, namely that originally obtained by Schonfeld [18], which however appeared to be in conflict with the BPS inequality for a central charge without quantum corrections.

Since this appeared to be a pure one-loop effect, Ref. [24] proposed the conjecture that it may be formulated in terms of a topological quantum anomaly. It was then shown by Shifman et al. [27], using a susy-preserving higher-derivative regularization method, that there is indeed an anomalous contribution to the central charge balancing the quantum corrections to the mass so that BPS saturation remains intact. In fact, it was later understood that multiplet shortening does in fact occur even in minimally susy $1+1$ dimensional theories, giving rise to single-state supermultiplets [28].

Both results, the nonvanishing mass correction and thus the necessity of a nonvanishing correction to the central charge, have been confirmed by a number of different methods $[29,30,25,31,32,33,26]$ validating also the finite mass formula in terms of only the discrete modes derived in Refs. [34, 35] based on the method of [36]. However, some authors claimed a nontrivial quantum correction to the central charge [29, 37] apparently without the need of the anomalous term proposed in Ref. [27].

In [38], we have shown that a particularly simple and elegant regularization scheme that yields the correct quantum mass of the susy kink is dimensional regularization, if the kink is embedded in higher dimensions as a domain wall [5]. Such a scheme was not considered before for the susy kink because both susy and the existence of finite-energy solutions seemed to tie one to one spatial dimension.

In [39] we then showed the $2+1$ dimensional domain wall is BPS saturated through a nontrivial quantum correction to the momentum in the extra dimension. This nontrivial correction is made possible by the fact that the $2+1$ dimensional theory spontaneously breaks parity, which also allows the appearance of domain wall fermions of only one chirality. By dimensionally reducing to $1+1$ dimensions, this parity-violating contribution to the extra momentum turns out to provide an anomalous contribution to the central charge as postulated in Ref. [27], thereby giving a novel physical explanation of the latter. This is in line with the well-known fact that central charges of susy theories can be reinterpreted as momenta in higher dimensions.

Hence, in the case of the susy kink, dimensional regularization is seen to be compatible with susy invariance only at the expense of a spontaneous parity violation, which in turn allows nonvanishing quantum corrections to the extra momentum in one higher spatial dimension. On the other hand, the surface term that usually exclusively provides the central charge does not receive quantum corrections in dimensional regularization, by the same reason that led to null results previously in other schemes [20, 21, 24]. The nontrivial anomalous quantum correction to the central charge operator is thus seen to be entirely the remnant of the spontaneous parity violation in the higher-dimensional theory in which a susy kink can be embedded by preserving minimal

\footnotetext{
${ }^{2}$ In mode regularization it turns out that one has to average over sets of boundary conditions to cancel both localized boundary energy and delocalized momentum $[25,26]$.
} 
susy.

\subsection{The model}

The real $\varphi^{4}$ model in $1+1$ dimensions with spontaneously broken $Z_{2}$ symmetry $(\varphi \rightarrow-\varphi)$ has topologically nontrivial finite-energy solutions called "kinks" which interpolate between the two degenerate vacuum states $\varphi= \pm v$. It has a minimally supersymmetric extension [40]

$$
\mathcal{L}=-\frac{1}{2}\left[\left(\partial_{\mu} \varphi\right)^{2}+U(\varphi)^{2}+\bar{\psi} \gamma^{\mu} \partial_{\mu} \psi+U^{\prime}(\varphi) \bar{\psi} \psi\right]
$$

where $\psi$ is a Majorana spinor, $\bar{\psi}=\psi^{\mathrm{T}} C$ with $C \gamma^{\mu}=$ $-\left(\gamma^{\mu}\right)^{T} C$. We shall use a Majorana representation of the Dirac matrices with $\gamma^{0}=-i \tau^{2}, \gamma^{1}=\tau^{3}$, and $C=\tau^{2}$ in terms of the standard Pauli matrices $\tau^{k}$ so that $\psi=\left(\begin{array}{c}\psi^{+} \\ \psi^{-}\end{array}\right)$ with real $\psi^{+}(x, t)$ and $\psi^{-}(x, t)$.

The $\varphi^{4}$ model is defined as the special case

$$
U(\varphi)=\sqrt{\frac{\lambda}{2}}\left(\varphi^{2}-v_{0}^{2}\right), \quad v_{0}^{2} \equiv \mu_{0}^{2} / \lambda
$$

where the $Z_{2}$ symmetry of the susy action also involves the fermions according to $\varphi \rightarrow-\varphi, \psi \rightarrow \gamma^{5} \psi$ with $\gamma^{5}=\gamma^{0} \gamma^{1}$. A classical kink at rest at $x=0$ which interpolates between the two vacua $\varphi= \pm v_{0}$ is given by [1]

$$
\varphi_{K}=v_{0} \tanh \left(\mu_{0} x / \sqrt{2}\right) .
$$

At the quantum level we have to renormalize, and we shall employ the simplest possible scheme ${ }^{3}$ which consists of putting all renormalization constants to unity except for a mass counterterm chosen such that tadpole diagrams cancel completely in the trivial vacuum. At the one-loop level and using dimensional regularization this gives

$$
\begin{aligned}
\delta \mu^{2} & =\lambda \delta v^{2}=\lambda \int \frac{d k_{0} d^{d} k}{(2 \pi)^{d+1}} \frac{-i}{k^{2}+m^{2}-i \epsilon} \\
& =\lambda \int \frac{d^{d} k}{(2 \pi)^{d}} \frac{1}{2\left[\vec{k}^{2}+m^{2}\right]^{1 / 2}},
\end{aligned}
$$

where $m=U^{\prime}(v)=\sqrt{2} \mu$ is the mass of elementary bosons and fermions and $k^{2}=\vec{k}^{2}-k_{0}^{2}$.

The susy invariance of the model under $\delta \varphi=\bar{\epsilon} \psi$ and $\delta \psi=(\not \partial \varphi-U) \epsilon$ (with $\mu_{0}^{2}$ replaced by $\mu^{2}+\delta \mu^{2}$ ) leads to the on-shell conserved Noether current

$$
j^{\mu}=-(\not \partial \varphi+U(\varphi)) \gamma^{\mu} \psi
$$

and two conserved charges $Q^{ \pm}=\int d x j^{0 \pm}$.

The model (2) is equally supersymmetric in $2+1$ dimensions, where we use $\gamma^{2}=\tau^{1}$. The same renormalization scheme can be used, only the renormalization constant (5) has to be evaluated for $d=2-\epsilon$ in place of $d=1-\epsilon$ spatial dimensions.

While classical kinks in $1+1$ dimensions have finite energy (rest mass) $M=m^{3} / \lambda$, in (noncompact) $2+1$ dimensions there exist no longer solitons of finite-energy. Instead one can have (one-dimensional) domain walls with a profile given by (4) which have finite surface (string) tension $M / L=m^{3} / \lambda$. With a compact extra dimension one can of course use these configurations to form "domain strings" of finite total energy proportional to the length $L$ of the string when wrapped around the extra dimension.

The $2+1$ dimensional case is different also with respect to the discrete symmetries of (2). In $2+1$ dimensions, $\gamma^{5}=\gamma^{0} \gamma^{1} \gamma^{2}= \pm \mathbf{1}$ corresponding to the two inequivalent choices available for $\gamma^{2}= \pm \tau^{1}$ (in odd space-time dimensions the Clifford algebra has two inequivalent irreducible representations). Therefore, the sign of the fermion mass (Yukawa) term can no longer be reversed by $\psi \rightarrow \gamma^{5} \psi$ and there is no longer the $Z_{2}$ symmetry $\varphi \rightarrow-\varphi, \psi \rightarrow \gamma^{5} \psi$.

What the $2+1$ dimensional model does break spontaneously is instead parity, which corresponds to changing the sign of one of the spatial coordinates. The Lagrangian is invariant under $x^{m} \rightarrow-x^{m}$ for a given spatial index $m=1,2$ together with $\varphi \rightarrow-\varphi$ (which thus is a pseudoscalar) and $\psi \rightarrow \gamma^{m} \psi$. Each of the trivial vacua breaks these invariances spontaneously, whereas a kink background in the $x^{1}$ direction with $\varphi_{K}\left(-x^{1}\right)=-\varphi_{K}\left(x^{1}\right)$ is symmetric with respect to $x^{1}$-reflections, but breaks $x^{2}=y$ reflection invariance.

\subsection{Susy algebra}

The susy algebra for the $1+1$ and the $2+1$ dimensional cases can both be covered by starting from $2+1$ dimensions, the $1+1$ dimensional case following from reduction by one spatial dimension.

In $2+1$ dimensions one obtains classically [41]

$$
\begin{aligned}
& \left\{Q^{\alpha}, \bar{Q}_{\beta}\right\}=2 i\left(\gamma^{M}\right)^{\alpha}{ }_{\beta} P_{M}, \quad(M=0,1,2) \\
& =2 i\left(\gamma^{0} H+\gamma^{1}\left(\tilde{P}_{x}+\tilde{Z}_{y}\right)+\gamma^{2}\left(\tilde{P}_{y}-\tilde{Z}_{x}\right)\right)^{\alpha}{ }_{\beta},(7)
\end{aligned}
$$

where we separated off two surface terms $\tilde{Z}_{m}$ in defining

$$
\begin{aligned}
& \tilde{P}_{m}=\int d^{d} x \tilde{\mathcal{P}}_{m}, \tilde{\mathcal{P}}_{m}=\dot{\varphi} \partial_{m} \varphi-\frac{1}{2}\left(\bar{\psi} \gamma^{0} \partial_{m} \psi\right), \\
& \tilde{Z}_{m}=\int d^{d} x \tilde{\mathcal{Z}}_{m}, \tilde{\mathcal{Z}}_{m}=U(\varphi) \partial_{m} \varphi=\partial_{m} W(\varphi)(9)
\end{aligned}
$$

with $W(\varphi) \equiv \int d \varphi U(\varphi)$.

Having a kink profile in the $x$-direction, which satisfies the Bogomolnyi equation $\partial_{x} \varphi_{K}=-U\left(\varphi_{K}\right)$, one finds that with our choice of Dirac matrices

$$
\begin{aligned}
& Q^{ \pm}=\int d^{2} x\left[\left(\dot{\varphi} \mp \partial_{y} \varphi\right) \psi^{ \pm}+\left(\partial_{x} \varphi \pm U(\varphi)\right) \psi^{\mp}\right], \\
& \left\{Q^{ \pm}, Q^{ \pm}\right\}=2\left(H \pm\left(\tilde{Z}_{x}-\tilde{P}_{y}\right)\right)
\end{aligned}
$$

and the charge $Q^{+}$leaves the topological (domain-wall) vacuum $\varphi=\varphi_{K}, \psi=0$ invariant. This corresponds to classical BPS saturation, since with $P_{x}=0$ and $\tilde{P}_{y}=0$ one has $\left\{Q^{+}, Q^{+}\right\}=2\left(H+\tilde{Z}_{x}\right)$ and, indeed, with a kink domain wall $\tilde{Z}_{x} / L^{d-1}=W(+v)-W(-v)=-M / L^{d-1}$.

\footnotetext{
${ }^{3}$ See [38] for a detailed discussion of more general renormalization schemes in this context.
} 
At the quantum level, hermiticity of $Q^{ \pm}$implies

$$
\langle s|H| s\rangle \geq\left|\left\langle s\left|P_{y}\right| s\right\rangle\right| \equiv\left|\left\langle s\left|\left(\tilde{P}_{y}-\tilde{Z}_{x}\right)\right| s\right\rangle\right| .
$$

This inequality is saturated when

$$
Q^{+}|s\rangle=0
$$

so that BPS states correspond to massless states $P_{M} P^{M}=0$ with $P_{y}=M$ for a kink domain wall in the $x$-direction, however with infinite momentum and energy unless the $y$ direction is compact with finite length $L$.

Classically, the susy algebra in $1+1$ dimensions is obtained from (7) simply by dropping $\tilde{P}_{y}$ as well as $\tilde{Z}_{y}$ so that $P_{x} \equiv \tilde{P}_{x}$. The term $\gamma^{2} \tilde{Z}_{x}$ remains, however, with $\gamma^{2}$ being the nontrivial $\gamma^{5}$ of $1+1$ dimensions. The susy algebra simplifies to

$$
\left\{Q^{ \pm}, Q^{ \pm}\right\}=2(H \pm Z), \quad\left\{Q^{+}, Q^{-}\right\}=2 P_{x}
$$

and one has the inequality

$$
\langle s|H| s\rangle \geq|\langle s|Z| s\rangle|
$$

for any quantum state $s$. BPS saturated states have $Q^{+}|s\rangle=$ 0 or $Q^{-}|s\rangle=0$, corresponding to kink and antikink, respectively, and preserve half of the supersymmetry.

\subsection{Fluctuations}

In a kink (or kink domain wall) background one spatial direction is singled out and we choose this to be along $x$. The direction orthogonal to the kink direction (parallel to the domain wall) will be denoted by $y$.

The quantum fields can then be expanded in the analytically known kink eigenfunctions [1] times plane waves in the extra dimensions. For the bosonic fluctuations we have $\left[-\square+\left(U^{\prime 2}+U U^{\prime \prime}\right)\right] \eta=0$ which is solved by

$$
\begin{aligned}
\eta=\int \frac{d^{d-1} \ell}{(2 \pi)^{\frac{d-1}{2}}} \sum \frac{d k}{\sqrt{4 \pi \omega}} & \left(a_{k, \ell} e^{-i(\omega t-\ell y)} \phi_{k}(x)\right. \\
& \left.+a_{k, \ell}^{\dagger} e^{i(\omega t-\ell y)} \phi_{k}^{*}(x)\right) .
\end{aligned}
$$

The kink eigenfunctions $\phi_{k}$ are normalized according to $\int d x|\phi|^{2}=1$ for the discrete states and to Dirac distributions for the continuum states according to $\int d x \phi_{k}^{*} \phi_{k^{\prime}}=$ $2 \pi \delta\left(k-k^{\prime}\right)$. The mode energies are $\omega=\sqrt{\omega_{k}^{2}+\ell^{2}}$ where $\omega_{k}$ is the energy in the 1+1-dimensional case.

The canonical equal-time commutation relations $\left[\eta(\vec{x}), \dot{\eta}\left(\vec{x}^{\prime}\right)\right]=i \delta\left(\vec{x}-\vec{x}^{\prime}\right)$ are fulfilled with

$$
\left[a_{k, \ell}, a_{k^{\prime}, \ell^{\prime}}^{\dagger}\right]=\delta_{k k^{\prime}} \delta\left(\ell-\ell^{\prime}\right)
$$

where for the continuum states $\delta_{k, k^{\prime}}$ becomes a Dirac delta.

For the fermionic modes which satisfy the Dirac equation $\left[\not \partial+U^{\prime}\right] \psi=0$ one finds

$$
\begin{aligned}
& \psi=\psi_{0}+ \\
& \int \frac{d^{d-1} \ell}{(2 \pi)^{\frac{d-1}{2}}} \sum \frac{d k}{\sqrt{4 \pi \omega}}\left[b_{k, \ell} e^{-i(\omega t-\ell y)}\left(\begin{array}{c}
\sqrt{\omega+\ell} \phi_{k}(x) \\
\sqrt{\omega-\ell} i s_{k}(x)
\end{array}\right)\right. \\
& \left.\left.+b_{k, \ell}^{\dagger} \text { (c.c.) }\right)\right]
\end{aligned}
$$

with

$$
\psi_{0}=\int \frac{d^{d-1} \ell}{(2 \pi)^{\frac{d-1}{2}}} b_{0, \ell} e^{-i \ell(t-y)}\left(\begin{array}{c}
\phi_{0} \\
0
\end{array}\right), \quad b_{0}^{\dagger}(\ell)=b_{0}(-\ell) .
$$

Thus, the fermionic zero mode ${ }^{4}$ of the susy kink turns into massless modes located on the domain wall, which have only one chirality, forming a Majorana-Weyl domain wall fermion $[38,7] .^{5}$

For the massive modes the Dirac equation relates the eigenfunctions appearing in the upper and the lower components of the spinors as follows:

$$
s_{k}=\frac{1}{\omega_{k}}\left(\partial_{x}+U^{\prime}\right) \phi_{k}=\frac{1}{\sqrt{\omega^{2}-\ell^{2}}}\left(\partial_{x}+U^{\prime}\right) \phi_{k}
$$

so that the function $s_{k}$ is the SUSY-quantum mechanical [42] partner state of $\phi_{k}$ and thus coincides with the eigen modes of the sine-Gordon model (hence the notation) [43]. With (20), their normalization is the same as that of the $\phi_{k}$.

The canonical equal-time anti-commutation relations $\left\{\psi^{\alpha}(\vec{x}), \psi^{\beta}\left(\vec{x}^{\prime}\right)\right\}=\delta^{\alpha \beta} \delta\left(\vec{x}-\vec{x}^{\prime}\right)$ are satisfied if

$$
\begin{aligned}
\left\{b_{0}(\ell), b_{0}^{\dagger}\left(\ell^{\prime}\right)\right\} & =\left\{b_{0}(\ell), b_{0}\left(-\ell^{\prime}\right)\right\}=\delta\left(\ell-\ell^{\prime}\right), \\
\left\{b_{k, \ell}, b_{k^{\prime}, \ell^{\prime}}^{\dagger}\right\} & =\delta_{k, k^{\prime}} \delta\left(\ell-\ell^{\prime}\right)
\end{aligned}
$$

and again the $\delta_{k, k^{\prime}}$ becomes a Dirac delta for the continuum states. The algebra (21) and the solution for the massless mode (18) show that the operator $b_{0}(\ell)$ creates right-moving massless states on the wall when $\ell$ is negative and annihilates them for positive momentum $\ell$. Thus only massless states with momentum in the positive $y$-direction can be created. Changing the representation of the gamma matrices by $\gamma^{2} \rightarrow-\gamma^{2}$, which is inequivalent to the original one, reverses the situation. Now only massless states with momenta in the positive $y$-direction exist. Thus depending on the representation of the Clifford algebra one chirality of the domain wall fermions is singled out. This is a reflection of the spontaneous violation of parity when embedding the susy kink as a domain wall in $2+1$ dimensions.

Notice that in (18) $d$ can be only 2 or 1 , for which $\ell$ has 1 or 0 components, so for strictly $d=1 \ell \equiv 0$. In order to have a susy-preserving dimensional regularization scheme by dimensional reduction, we shall start from $d=2$ spatial dimensions, and then make $d$ continuous and smaller than 2 .

\footnotetext{
${ }^{4}$ By a slight abuse of notation we shall always label this by a subscript 0 , but this should not be confused with the threshold mode $k=0$ (which does not appear explicitly anywhere below).

${ }^{5}$ The mode with $\ell=0$ corresponds in $1+1$ dimensions to the zero mode of the susy kink. It has to be counted as half a degree of freedom in mode regularization [25]. For dimensional regularization such subtleties do not play a role because the zero mode only gives scaleless integrals and these vanish.
} 


\subsection{Energy corrections}

Using the mode expansions in the Hamiltonian expanded to second order in quantum fluctuations, one finds that the bosonic and fermionic contributions combine into

$$
\begin{aligned}
& \int d x d^{d-1} y\left\langle\mathcal{H}^{(2)}\right\rangle \\
& =\frac{L^{d-1}}{2} \int d x \int \frac{d^{d-1} \ell}{(2 \pi)^{d-1}} \sum \frac{d k}{2 \pi} \frac{\omega}{2}\left(\left|\phi_{k}\right|^{2}-\left|s_{k}\right|^{2}\right) .
\end{aligned}
$$

In these expressions, the massless modes (which correspond to the zero mode of the 1+1 dimensional kink) can be dropped in dimensional regularization as scaleless and thus vanishing contributions, and the massive discrete modes cancel between bosons and fermions. ${ }^{6}$ Carrying out the $x$ integration over the continuous mode functions gives a difference of spectral densities, namely

$$
\int d x\left(\left|\phi_{k}(x)\right|^{2}-\left|s_{k}(x)\right|^{2}\right)=-\theta^{\prime}(k)=-\frac{2 m}{k^{2}+m^{2}},
$$

where $\theta(k)$ is the additional phase shift of the mode functions $s_{k}$ compared to $\phi_{k}$.

Combining (22) and (23), and adding in the counterterm contribution from (5) leads to a simple integral

$$
\begin{aligned}
\frac{\Delta M^{(1)}}{L^{d-1}} & =-\frac{1}{4} \int \frac{d k d^{d-1} \ell}{(2 \pi)^{d}} \omega \theta^{\prime}(k)+m \delta v^{2} \\
& =-\frac{1}{4} \int \frac{d k d^{d-1} \ell}{(2 \pi)^{d}} \frac{\ell^{2}}{\omega} \theta^{\prime}(k) \\
& =-\frac{2}{d} \frac{\Gamma\left(\frac{3-d}{2}\right)}{(4 \pi)^{\frac{d+1}{2}}} m^{d}
\end{aligned}
$$

This reproduces the correct known result for the susy kink mass correction $\Delta M^{(1)}=-m /(2 \pi)$ (for $d=1$ ) and the surface (string) tension of the $2+1$ dimensional susy kink domain wall $\Delta M^{(1)} / L=-m^{2} /(8 \pi)$ (for $d=2$ ) [38].

\subsection{Anomalous contributions to the central charge}

In a kink (domain wall) background with only nontrivial $x$ dependence, the central charge density $\tilde{\mathcal{Z}}_{x}$ receives nontrivial contributions. Expanding $\tilde{\mathcal{Z}}_{x}$ around the kink background gives

$$
\begin{aligned}
\tilde{\mathcal{Z}}_{x}= & U \partial_{x} \varphi_{K}-\frac{\delta \mu^{2}}{\sqrt{2 \lambda}} \partial_{x} \varphi_{K}+\partial_{x}(U \eta)+\frac{1}{2} \partial_{x}\left(U^{\prime} \eta^{2}\right) \\
& +O\left(\eta^{3}\right) .
\end{aligned}
$$

Again only the part quadratic in the fluctuations contributes to the integrated quantity at one-loop order ${ }^{7}$. However, using $U^{\prime}(x= \pm \infty)= \pm m$ this leads just to the contribution

$$
\begin{aligned}
& \int d x\left\langle\frac{1}{2} \partial_{x}\left(U^{\prime} \eta^{2}\right)\right\rangle=\left.\frac{1}{2} U^{\prime}\left\langle\eta^{2}\right\rangle\right|_{-\infty} ^{\infty} \\
& =m \int \frac{d^{d-1} \ell}{(2 \pi)^{d-1}} \int \frac{d k}{2 \pi} \frac{1}{2 \omega} \equiv m \delta v^{2}
\end{aligned}
$$

which matches precisely the counterterm $\propto \delta \mu^{2}$ from requiring vanishing tadpoles. Straightforward application of the rules of dimensional regularization thus leads to a null result for the net one-loop correction to $\left\langle\tilde{Z}_{x}\right\rangle$ in the same way as found in Refs. [20, 21, 24] in other schemes.

On the other hand, by considering the less singular combination $\left\langle H+\tilde{Z}_{x}\right\rangle$ and showing that it vanishes exactly, it was concluded in Ref. [29] that $\left\langle\tilde{Z}_{x}\right\rangle$ has to compensate any nontrivial result for $\langle H\rangle$, which in Ref. [29] was obtained by subtracting successive Born approximations for scattering phase shifts. In fact, Ref. [29] explicitly demonstrates how to rewrite $\left\langle\tilde{Z}_{x}\right\rangle$ into $-\langle H\rangle$, apparently without the need for the anomalous terms in the quantum central charge operator derived in Ref. [27].

The resolution of this discrepancy is that Ref. [29] did not regularize $\left\langle\tilde{Z}_{x}\right\rangle$ and the manipulations needed to rewrite it as $-\langle H\rangle$ (which eventually is regularized and renormalized) are ill-defined. Using dimensional regularization one in fact obtains a nonzero result for $\left\langle H+\tilde{Z}_{x}\right\rangle$, apparently in violation of susy.

However, dimensional regularization by embedding the kink as a domain wall in (up to) one higher dimension, which preserves susy, instead leads to

$$
\left\langle H+\tilde{Z}_{x}-\tilde{P}_{y}\right\rangle=0
$$

i.e. the saturation of (12), as we shall now verify.

The bosonic contribution to $\left\langle\tilde{P}_{y}\right\rangle$ involves

$$
\frac{1}{2}\left\langle\dot{\eta} \partial_{y} \eta+\partial_{y} \eta \dot{\eta}\right\rangle=-\int \frac{d^{d-1} \ell}{(2 \pi)^{d-1}} \sum \frac{d k}{2 \pi} \frac{\ell}{2}\left|\phi_{k}(x)\right|^{2} .
$$

The $\ell$-integral factorizes and gives zero both because it is a scale-less integral and because the integrand is odd in $\ell$. Only the fermions turn out to give interesting contributions:

$$
\begin{aligned}
\left\langle\tilde{\mathcal{P}}_{y}\right\rangle= & \frac{i}{2}\left\langle\psi^{\dagger} \partial_{y} \psi\right\rangle \\
= & \frac{1}{2} \int \frac{d^{d-1} \ell}{(2 \pi)^{d-1}} \sum \frac{d k}{2 \pi} \frac{\ell}{2 \omega}\left[(\omega+\ell)\left|\phi_{k}\right|^{2}\right. \\
& \left.+(\omega-\ell)\left|s_{k}\right|^{2}\right] \\
= & \frac{1}{2} \int \frac{d^{d-1} \ell}{(2 \pi)^{d-1}} \ell \theta(-\ell)\left|\phi_{0}\right|^{2}+ \\
& +\frac{1}{2} \int \frac{d^{d-1} \ell}{(2 \pi)^{d-1}} \sum \frac{d k}{2 \pi}\left(\frac{\ell}{2}\left(\left|\phi_{k}\right|^{2}+\left|s_{k}\right|^{2}\right)\right. \\
& \left.+\frac{\ell^{2}}{2 \omega}\left(\left|\phi_{k}\right|^{2}-\left|s_{k}\right|^{2}\right)\right) .
\end{aligned}
$$

\footnotetext{
${ }^{6}$ The zero mode contributions in fact do not cancel by themselves between bosons and fermions, because the latter are chiral. This noncancellation is in fact crucial in energy cutoff regularization (see Ref. [38]).

${ }^{7}$ Again, this does not hold for the central charge density locally [27, 31].
} 
From the last sum-integral we have separated off the contribution of the zero mode of the kink, which turns into chiral domain wall fermions for $d>1$. The contribution of the latter no longer vanishes by symmetry, but the $\ell$-integral is still scale-less and therefore put to zero in dimensional regularization. The first sum-integral on the right-hand side is again zero by both symmetry and scalelessness, but the final term is not. The $\ell$-integration no longer factorizes because $\omega=\sqrt{k^{2}+\ell^{2}+m^{2}}$. Integrating over $x$ and using (23) one in fact obtains exactly the same expression as in the one-loop result for the energy, Eq. (24).

So for all $d \leq 2$ we have BPS saturation, $\langle H\rangle=$ $\left|\left\langle\tilde{Z}_{x}-\tilde{P}_{y}\right\rangle\right|$, which in the limit $d \rightarrow 1$, the susy kink, is made possible by a nonvanishing $\left\langle\tilde{P}_{y}\right\rangle$. The anomaly in the central charge is seen to arise from a parity-violating contribution in $d=1+\epsilon$ dimensions which is the price to be paid for preserving supersymmetry when going up in dimensions to embed the susy kink as a domain wall.

It is perhaps worth emphasizing that the above results do not depend on the details of the spectral densities associated with the mode functions $\phi_{k}$ and $s_{k}$. In the integrated quantities $\langle H\rangle$ and $\left\langle\tilde{P}_{y}\right\rangle$ only the difference of the spectral densities as given by (23) is responsible for the nonvanishing contribution. The function $\theta(k)$ therein is entirely fixed by the form of the Dirac equation in the asymptotic regions $x \rightarrow \pm \infty$ far away from the kink [21].

\subsection{Dimensional reduction and evanescent counterterms}

We now describe how the central charge anomaly can be recovered from Siegel's version of dimensional regularization [6] where $n$ is smaller than the dimension of spacetime and where one keeps the number of field components fixed, but lowers the number of coordinates and momenta from 2 to $n<2$. At the one-loop level one encounters 2-dimensional $\delta_{\mu}^{\nu}$ coming from Dirac matrices, and $n$-dimensional $\hat{\delta}_{\mu}^{\nu}$ from loop momenta. An important concept which is going to play a role are the evanescent counterterms [14] involving the factor $\frac{1}{\epsilon} \hat{\hat{\delta}}_{\mu}^{\nu} \gamma_{\nu} \psi$, where $\hat{\hat{\delta}}_{\mu}^{\nu} \equiv \delta_{\mu}^{\nu}-\hat{\delta}_{\mu}^{\nu}$ has only $\epsilon=2-n$ nonvanishing components.

In the trivial vacuum, expanding the supercurrent $j_{\mu}=$ $-(\not \partial \varphi+U(\varphi)) \gamma_{\mu} \psi$ into quantum fields yields

$j_{\mu}=-\left(\not \partial \eta+U^{\prime}(v) \eta+\frac{1}{2} U^{\prime \prime}(v) \eta^{2}\right) \gamma_{\mu} \psi+\frac{1}{\sqrt{2 \lambda}} \delta \mu^{2} \gamma_{\mu} \psi$.

Only matrix elements with one external fermion are divergent. The term involving $U^{\prime \prime}(v) \eta^{2}$ in (30) gives rise to a divergent scalar tadpole that is cancelled completely by the counterterm $\delta \mu^{2}$ (which itself is due to an $\eta$ and a $\psi$ loop). The only other divergent diagram is due to the term involving $\not \partial \eta$ in (30) and has the form a $\psi$-selfenergy. Its singular part reads

$$
\begin{aligned}
& \left\langle 0\left|j_{\mu}\right| p\right\rangle^{\operatorname{div}} \\
= & i U^{\prime \prime}(v) \int_{0}^{1} d x \int \frac{d^{n} \kappa}{(2 \pi)^{n}} \frac{\not k \gamma_{\mu} \not k u(p)}{\left[\kappa^{2}+p^{2} x(1-x)+m^{2}\right]^{2}} .
\end{aligned}
$$

Using $\hat{\delta}_{\mu}^{\nu} \equiv \delta_{\mu}^{\nu}-\hat{\hat{\delta}}_{\mu}^{\nu}$ we find that under the integral

$$
\not \gamma_{\mu} \kappa \kappa=-\kappa^{2}\left(\delta_{\mu}^{\lambda}-\frac{2}{n} \hat{\delta}_{\mu}^{\lambda}\right) \gamma_{\lambda}=\frac{\epsilon}{n} \kappa^{2} \gamma_{\mu}-\frac{2}{n} \kappa^{2} \hat{\hat{\delta}}_{\mu}^{\lambda} \gamma_{\lambda}
$$

so that

$$
\left\langle 0\left|j_{\mu}\right| p\right\rangle^{\operatorname{div}}=\frac{U^{\prime \prime}(v)}{2 \pi} \frac{\hat{\hat{\delta}}_{\mu}^{\lambda}}{\epsilon} \gamma_{\lambda} u(p) .
$$

Hence, the regularized one-loop contribution to the susy current contains the evanescent operator

$$
j_{\mu}^{\operatorname{div}}=\frac{U^{\prime \prime}(\varphi)}{2 \pi} \frac{\hat{\hat{\delta}}_{\mu}^{\lambda}}{\epsilon} \gamma_{\lambda} \psi
$$

This is by itself a conserved quantity, because all fields depend only on the $n$-dimensional coordinates, but it has a nonvanishing contraction with $\gamma^{\mu}$. The latter gives rise to an anomalous contribution to the renormalized conformalsusy current $\not \not j_{\mu}^{\text {ren. }}$ where $j_{\mu}^{\text {ren. }}=j_{\mu}-j_{\mu}^{d i v}$,

$$
\partial^{\mu}\left(\not x j_{\mu}^{\text {ren. }}\right)_{\text {anom. }}=-\gamma^{\mu} j_{\mu}^{\text {div }}=-\frac{U^{\prime \prime}}{2 \pi} \psi
$$

(There are also nonvanishing nonanomalous contributions to $\partial^{\mu}\left(\not x j_{\mu}\right)$ because our model is not conformal-susy invariant at the classical level [44].)

Ordinary susy on the other hand is unbroken; there is no anomaly in the divergence of $j_{\mu}^{\text {ren. }}$. A susy variation of $j_{\mu}$ involves the energy-momentum tensor and the topological central-charge current $\zeta_{\mu}$ according to

$$
\delta j_{\mu}=-2 T_{\mu}{ }^{\nu} \gamma_{\nu} \epsilon-2 \zeta_{\mu} \gamma^{5} \epsilon
$$

where classically $\zeta_{\mu}=\epsilon_{\mu \nu} U \partial^{\nu} \varphi$.

At the quantum level, the counter-term $j_{\mu}^{\text {ct }}=-j_{\mu}^{\text {div. in- }}$ duces an additional contribution to the central charge current

$$
\zeta_{\mu}^{\mathrm{anom}}=\frac{1}{4 \pi} \frac{\hat{\hat{\delta}}_{\mu}^{\nu}}{\epsilon} \epsilon_{\nu \rho} \partial^{\rho} U^{\prime}
$$

which despite appearances is a finite quantity: using that total antisymmetrization of the three lower indices has to vanish in two dimensions gives

$$
\hat{\hat{\delta}}_{\mu}^{\nu} \epsilon_{\nu \rho}=\epsilon \epsilon_{\mu \rho}+\hat{\hat{\delta}}_{\rho}^{\nu} \epsilon_{\nu \mu}
$$

and together with the fact the $U^{\prime}$ only depends on $n$ dimensional coordinates this finally yields

$$
\zeta_{\mu}^{\mathrm{anom}}=\frac{1}{4 \pi} \epsilon_{\mu \rho} \partial^{\rho} U^{\prime}
$$

in agreement with the anomaly in the central charge as obtained previously. 


\section{The (susy) vortex.}

We next considered [45] the Abrikosov-Nielsen-Olesen [46, $47,48,49]$ vortex solution of the abelian Higgs model in $2+1$ dimensions which has a supersymmetric extension $[50,51]$ (see also [52, 53]) such that classically the Bogomolnyi bound [54] is saturated. We employed our variant of dimensional regularization to the $N=2$ vortex by dimensionally reducing the $N=1$ abelian Higgs model in $3+1$ dimensions. We confirmed the results of $[50,55,56]$ that in a particular gauge (background-covariant Feynman-'t Hooft) the sums over zero-point energies of fluctuations in the vortex background cancel completely, but contrary to $[50,55]$ we found a nonvanishing quantum correction to the vortex mass coming from a finite renormalization of the expectation value of the Higgs field in this gauge $[57,56]$. In contrast to [50], where a null result for the quantum corrections to the central charge was stated, we show that the central charge receives also a net nonvanishing quantum correction, namely from a nontrivial phase in the fluctuations of the Higgs field in the vortex background, which contributes to the central charge even though the latter is a surface term that can be evaluated far away from the vortex. The correction to the central charge exactly matches the correction to the mass of the vortex.

In Ref. [55], it was claimed that the usual multiplet shortening arguments in favor of BPS saturation would not be applicable to the $N=2$ vortex since in the vortex background there would be two rather than one fermionic zero modes [58], leading to two short multiplets which have the same number of states as one long multiplet. ${ }^{8}$ We showed however that the extra zero mode postulated in [55] has to be discarded because its gaugino component is singular, and that only after doing so there is agreement with the results from index theorems $[59,58,60]$. For this reason, standard multiplet shortening arguments do apply, explaining the BPS saturation at the quantum level that we observe in our explicit one-loop calculations.

The $N=2$ susy vortex in $2+1$ dimensions is the solitonic (finite-energy) solution of the abelian Higgs model which can be obtained by dimensional reduction from a 3+1-dimensional $N=1$ model. We shall use the latter for the purpose of dimensional regularization of the 2+1dimensional model by susy-preserving dimensional reduction from $3+1$ dimensions (where the vortex has infinite mass but finite energy-density).

\subsection{The model}

The superspace action for the vortex in terms of 3+1dimensional superfields contains an $N=1$ abelian vector multiplet and an $N=1$ scalar multiplet, coupled as usual, together with a Fayet-Iliopoulos term but without superpo- tential,

$$
\mathcal{L}=\int d^{2} \theta W^{\alpha} W_{\alpha}+\int d^{4} \theta \bar{\Phi} e^{e V} \Phi+\kappa \int d^{4} \theta V .
$$

In terms of 2-component spinors in 3+1 dimensions, the action reads ${ }^{9}$

$$
\begin{aligned}
\mathcal{L}= & -\frac{1}{4} F_{\mu \nu}^{2}+\bar{\chi}^{\dot{\alpha}} i \bar{\sigma}_{\dot{\alpha} \beta}^{\mu} \partial_{\mu} \chi^{\beta}+\frac{1}{2} D^{2}+\left(\kappa-e|\phi|^{2}\right) D \\
& -\left|D_{\mu} \phi\right|^{2}+\bar{\psi}^{\dot{\alpha}} i \bar{\sigma}_{\dot{\alpha} \beta}^{\mu} D_{\mu} \psi^{\beta}+|F|^{2} \\
& +\sqrt{2} e\left[\phi^{*} \chi_{\alpha} \psi^{\alpha}+\phi \bar{\chi}_{\dot{\alpha}} \bar{\psi}^{\dot{\alpha}}\right],
\end{aligned}
$$

where $D_{\mu}=\partial_{\mu}-i e A_{\mu}$ when acting on $\phi$ and $\psi$, and $F_{\mu \nu}=\partial_{\mu} A_{\nu}-\partial_{\nu} A_{\mu}$. Elimination of the auxiliary field $D$ yields the scalar potential $\mathcal{V}=\frac{1}{2} D^{2}=\frac{1}{2} e^{2}\left(|\phi|^{2}-v^{2}\right)^{2}$ with $v^{2} \equiv \kappa / e$.

In $3+1$ dimensions, this model has a chiral anomaly, and in order to cancel the chiral U(1) anomaly, additional scalar multiplets would be needed such that the sum over charges vanishes, $\sum_{i} e_{i}=0$.

In $2+1$ dimensions, dimensional reduction gives an $N=$ 2 model involving, in the notation of [55], a real scalar $N=A_{3}$ and two complex (Dirac) spinors $\psi=\left(\psi^{\alpha}\right)$, $\chi=\left(\chi^{\alpha}\right)$.

Completing squares in the bosonic part of the classical Hamiltonian density one finds the Bogomolnyi equations and the central charge

$$
\begin{aligned}
\mathcal{H}= & \frac{1}{4} F_{k l}^{2}+\left|D_{k} \phi\right|^{2}+\frac{1}{2} e^{2}\left(|\phi|^{2}-v^{2}\right)^{2} \\
= & \frac{1}{2}\left|D_{k} \phi+i \epsilon_{k l} D_{l} \phi\right|^{2}+\frac{1}{2}\left(F_{12}+e\left(|\phi|^{2}-v^{2}\right)\right)^{2} \\
& +\frac{e}{2} v^{2} \epsilon_{k l} F_{k l}-i \partial_{k}\left(\epsilon_{k l} \phi^{*} D_{l} \phi\right)
\end{aligned}
$$

where $k, l$ are the spatial indices in $2+1$ dimensions. The classical central charge reads

$$
Z=\int d^{2} x \epsilon_{k l} \partial_{k}\left(e v^{2} A_{l}-i \phi^{*} D_{l} \phi\right),
$$

where asymptotically $D_{l} \phi$ tends to zero exponentially fast. Classically, BPS saturation $E=|Z|=2 \pi v^{2} n$ holds when the BPS equations $\left(D_{1} \pm i D_{2}\right) \phi \equiv D_{ \pm} \phi=0$ and $F_{12} \pm e\left(|\phi|^{2}-v^{2}\right)=0$ are satisfied, where the upper and lower sign corresponds to vortex and antivortex, respectively. The vortex solution with winding number $n$ is given by $\left(A_{ \pm}^{\mathrm{V}} \equiv A_{1}^{\mathrm{V}} \pm i A_{2}^{\mathrm{V}}\right)$

$$
\phi_{\mathrm{V}}=e^{i n \theta} f(r), \quad e A_{+}^{\mathrm{V}}=-i e^{i \theta} \frac{a(r)-n}{r},
$$

where $f^{\prime}(r)=\frac{a}{r} f(r)$ and $a^{\prime}(r)=r e^{2}\left(f(r)^{2}-v^{2}\right)$ with boundary conditions [49]

$$
\begin{array}{cl}
a(r \rightarrow \infty)=0, & f(r \rightarrow \infty)=v, \\
a(r \rightarrow 0)=n+O\left(r^{2}\right), & f(r \rightarrow 0) \propto r^{n}+O\left(r^{n+2}\right) .
\end{array}
$$

\footnotetext{
${ }^{8}$ Incidentally, Refs. [55, 58] considered supersymmetric Maxwell-Chern-Simons theory, which contains the supersymmetric abelian Higgs model as a special case.

${ }^{9}$ Our conventions are $\eta^{\mu \nu}=(-1,+1,+1,+1), \chi^{\alpha}=\epsilon^{\alpha \beta} \chi_{\beta}$ and $\bar{\chi}^{\dot{\alpha}}=\epsilon^{\dot{\alpha} \dot{\beta}} \bar{\chi}_{\dot{\beta}}$ with $\epsilon^{\alpha \beta}=\epsilon_{\alpha \beta}=-\epsilon^{\dot{\alpha} \dot{\beta}}=-\epsilon_{\dot{\alpha} \dot{\beta}}$ and $\epsilon^{12}=+1$. In particular we have $\bar{\psi}_{\dot{\alpha}}=\left(\psi_{\alpha}\right)^{*}$ but $\bar{\psi}^{\dot{\alpha}}=-\left(\psi^{\alpha}\right)^{*}$. Furthermore, $\bar{\sigma}^{\mu}=(-\mathbf{1}, \tilde{\sigma})$ with the usual representation for the Pauli matrices $\vec{\sigma}$.
} 


\subsection{Fluctuation equations}

For the calculation of quantum corrections to a vortex solution we decompose $\phi$ into a classical background part $\phi_{\mathrm{V}}$ and a quantum part $\eta$. Similarly, $A_{\mu}$ is decomposed as $A_{\mu}^{\mathrm{V}}+a_{\mu}$, where only $A_{\mu}^{\mathrm{V}}$ with $\mu=1,2$ is nonvanishing. We use a background $R_{\xi}$ [61] gauge fixing term which is quadratic in the quantum gauge fields,

$$
\mathcal{L}_{\text {g.fix }}=-\frac{1}{2 \xi}\left(\partial_{\mu} a^{\mu}-i e \xi\left(\phi_{\mathrm{V}} \eta^{*}-\phi_{\mathrm{V}}^{*} \eta\right)\right)^{2} .
$$

The corresponding Faddeev-Popov Lagrangian reads

$$
\mathcal{L}_{\text {ghost }}=b\left(\partial_{\mu}^{2}-e^{2} \xi\left\{2\left|\phi_{\mathrm{V}}\right|^{2}+\phi_{\mathrm{V}} \eta^{*}+\phi_{\mathrm{V}}^{*} \eta\right\}\right) c .
$$

The fluctuation equations in $2+1$ dimensions have been given in $[50,55]$ for the choice $\xi=1$ (Feynman- 't Hooft gauge) which leads to important simplifications. We shall mostly use this gauge choice when considering fluctuations in the solitonic background, but will carry out renormalization in the trivial vacuum for general $\xi$ to exhibit some of the intermediate gauge dependences.

Because we are going to consider dimensional regularization by dimensional reduction from the $3+1$ dimensional model, we shall need the form of the fluctuation equations with derivatives in the $x^{3}$ direction included. (This one trivial extra dimension will eventually be turned into $\epsilon \rightarrow 0$ dimensions.)

In the 't Hooft-Feynman gauge, the part of the bosonic action quadratic in the quantum fields reads

$$
\begin{aligned}
\mathcal{L}_{\text {bos }}^{(2)}= & -\frac{1}{2}\left(\partial_{\mu} a_{\nu}\right)^{2}-e^{2}\left|\phi_{\mathrm{V}}\right|^{2} a_{\mu}^{2} \\
& -\left|D_{\mu}^{\mathrm{V}} \eta\right|^{2}-e^{2}\left(3\left|\phi_{\mathrm{V}}\right|^{2}-v^{2}\right)|\eta|^{2} \\
& -2 i e a^{\mu}\left[\eta^{*} D_{\mu}^{\mathrm{V}} \phi_{\mathrm{V}}-\eta\left(D_{\mu}^{\mathrm{V}} \phi_{\mathrm{V}}\right)^{*}\right] .
\end{aligned}
$$

In the trivial vacuum, which corresponds to $\phi_{\mathrm{V}} \rightarrow v$ and $A_{\mu}^{\mathrm{V}} \rightarrow 0$, the last term vanishes, but in the solitonic vacuum it couples the linearized field equations for the fluctuations $B \equiv\left(\eta, a_{+} / \sqrt{2}\right)$ with $a_{+}=a_{1}+i a_{2}$ to each other according to $(k=1,2)$

$$
\begin{aligned}
& \left(\partial_{3}^{2}-\partial_{t}^{2}\right) B \\
& =\left(\begin{array}{cc}
-\left(D_{k}^{\mathrm{V}}\right)^{2}+e^{2}\left(3\left|\phi_{\mathrm{V}}\right|^{2}-v^{2}\right) & i \sqrt{2} e\left(D_{-} \phi_{\mathrm{V}}\right) \\
-i \sqrt{2} e\left(D_{-} \phi_{\mathrm{V}}\right)^{*} & -\partial_{k}^{2}+2 e^{2}\left|\phi_{\mathrm{V}}\right|^{2}
\end{array}\right) B .
\end{aligned}
$$

The quartet $\left(a_{3}, a_{0}, b, c\right)$ with $b, c$ the Faddeev-Popov ghost fields has diagonal field equations at the linearized level

$$
\left(\partial_{\mu}^{2}-2 e^{2}\left|\phi_{\mathrm{V}}\right|^{2}\right) Q=0, \quad Q \equiv\left(a_{3}, a_{0}, b, c\right) .
$$

For the fermionic fluctuations, which we group as $U=$ $\left(\begin{array}{c}\psi^{1} \\ \bar{\chi}^{1}\end{array}\right), V=\left(\begin{array}{c}\psi^{2} \\ \bar{\chi}^{2}\end{array}\right)$, the linearized field equations read

$$
L U=i\left(\partial_{t}+\partial_{3}\right) V, \quad L^{\dagger} V=i\left(\partial_{t}-\partial_{3}\right) U,
$$

with

$$
\begin{aligned}
L & =\left(\begin{array}{cc}
i D_{+}^{\mathrm{V}} & \sqrt{2} e \phi_{\mathrm{V}} \\
-\sqrt{2} e \phi_{\mathrm{V}}^{*} & i \partial_{-}
\end{array}\right), \\
L^{\dagger} & =\left(\begin{array}{cc}
i D_{-}^{\mathrm{V}} & -\sqrt{2} e \phi_{\mathrm{V}} \\
\sqrt{2} e \phi_{\mathrm{V}}^{*} & i \partial_{+}
\end{array}\right) .
\end{aligned}
$$

Iteration shows that $U$ satisfies the same second order equations as the bosonic fluctuations $B$,

$$
\begin{aligned}
& L^{\dagger} L U=\left(\partial_{3}^{2}-\partial_{t}^{2}\right) U, \quad L^{\dagger} L B=\left(\partial_{3}^{2}-\partial_{t}^{2}\right) B \\
& L L^{\dagger} V=\left(\partial_{3}^{2}-\partial_{t}^{2}\right) V,
\end{aligned}
$$

with $L^{\dagger} L$ given by (48), whereas $V$ is governed by a diagonal equation with

$$
L L^{\dagger}=\left(\begin{array}{cc}
-\left(D_{k}^{\mathrm{V}}\right)^{2}+e^{2}\left|\phi_{\mathrm{V}}\right|^{2}+e^{2} v^{2} & 0 \\
0 & -\partial_{k}^{2}+2 e^{2}\left|\phi_{\mathrm{V}}\right|^{2}
\end{array}\right) .
$$

(In deriving these fluctuation equations we used the BPS equations throughout.)

\subsection{Renormalized mass}

At the classical level, the energy and central charge of vortices are multiples of $2 \pi v^{2}$ with $v^{2}=\kappa / e$. Renormalization of tadpoles, even when only by finite amounts, will therefore contribute directly to the quantum mass and central charge of the $N=2$ vortex, a fact that has been overlooked in the original literature $[50,55]$ on quantum corrections to the $N=2$ vortex. ${ }^{10}$

Adopting a "minimal" renormalization scheme where the scalar wave function renormalization constant $Z_{\phi}=1$, the renormalization of $v^{2}$ is fixed by the requirement of vanishing tadpoles in the trivial sector of the $2+1$ dimensional model. The calculation can be conveniently performed by using dimensional regularization of the $3+1$ dimensional $N=1$ model. For the calculation of the tadpoles we decompose $\phi=v+\eta \equiv v+(\sigma+i \rho) / \sqrt{2}$, where $\sigma$ is the Higgs field and $\rho$ the would-be Goldstone boson. The gauge fixing term (45) avoids mixed $a_{\mu}-\rho$ propagators, but there are mixed $\chi-\psi$ propagators, which can be diagonalized by introducing new spinors $s=(\psi+i \chi) / \sqrt{2}$ and $d=(\psi-i \chi) / \sqrt{2}$ with mass terms $m\left(s_{\alpha} s^{\alpha}-d_{\alpha} d^{\alpha}\right)+h . c$., where $m=\sqrt{2} e v$.

The part of the interaction Lagrangian which is relevant for $\sigma$ tadpoles to one-loop order is given by

$$
\begin{aligned}
\mathcal{L}_{\sigma-\text { tadpoles }}^{\text {int }}= & e\left(\chi_{\alpha} \psi^{\alpha}+\bar{\chi}_{\dot{\alpha}} \bar{\psi}^{\dot{\alpha}}\right) \sigma-\frac{e m}{2}\left(\sigma^{2}+\rho^{2}\right) \sigma \\
& -e m\left(a_{\mu}^{2}+\xi b c-\delta v^{2}\right) \sigma,
\end{aligned}
$$

where $b$ and $c$ are the Faddeev-Popov fields.

The one-loop contributions to the $\sigma$ tadpole thus read

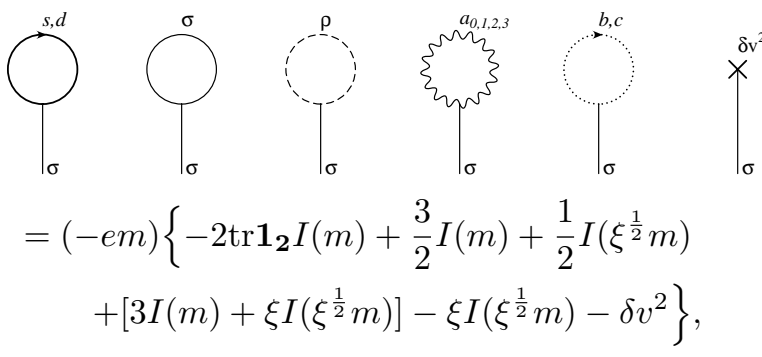

\footnotetext{
${ }^{10}$ The nontrivial renormalization of $\kappa / e$ has however been included in $[57,56]$.
} 
where

$$
\begin{aligned}
I(m) & =\int \frac{d^{3+\epsilon} k}{(2 \pi)^{3+\epsilon}} \frac{-i}{k^{2}+m^{2}} \\
& =-\frac{m^{1+\epsilon}}{(4 \pi)^{1+\epsilon / 2}} \frac{\Gamma\left(-\frac{1}{2}-\frac{\epsilon}{2}\right)}{\Gamma\left(-\frac{1}{2}\right)}=-\frac{m}{4 \pi}+O(\epsilon)
\end{aligned}
$$

Requiring that the sum of tadpole diagrams (56) vanishes fixes $\delta v^{2}$,

$$
\delta v^{2}=\left.\frac{1}{2}\left(I(m)+I\left(\xi^{\frac{1}{2}} m\right)\right)\right|_{D=3}=-\frac{1+\xi^{\frac{1}{2}}}{8 \pi} m .
$$

Because in dimensional regularization there are no poles in odd dimensions at the one-loop level, the result for $\delta v^{2}$ is finite, but it is nonvanishing.

As it turns out, this is the only contribution to the oneloop mass correction of the vortex. In the $\xi=1$ gauge the zero-point energies of the quartet $\left(a_{3}, a_{0}, b, c\right)$ cancels, and one is left with

$$
\frac{1}{2} \sum \omega_{\mathrm{bos}}-\frac{1}{2} \sum \omega_{\mathrm{ferm}}=\sum \omega_{U}-\sum \omega_{V},
$$

Using dimensional regularization these sums can be made well defined by replacing all eigen frequencies $\omega_{k}$ in $2+1$ dimensions by $\omega_{k, \ell}=\left(\omega_{k}^{2}+\ell^{2}\right)^{1 / 2}$ where $\ell$ are the extra momenta. Using index theorems, it has been shown that the spectral densities for $U$ and $V$ are equal up to zero modes $[50,55]$, and zero modes (massless modes upon embedding) do not contribute in dimensional regularization. Hence, $\sum \omega_{U}-\sum \omega_{V}=0$, as we have also verified more directly [16], and the only nonvanishing quantum correction of the vortex mass is from renormalization. In our "minimal" renormalization scheme we thus have

$$
E=2 \pi|n|\left(v^{2}+\left.\delta v^{2}\right|_{\xi=1}\right)=2 \pi|n|\left(v^{2}-\frac{m}{4 \pi}\right) .
$$

\subsection{Central charge}

By starting from the susy algebra in $3+1$ dimensions one can derive the central charge in $2+1$ dimensions as the component $T_{03}$ of

$$
T^{\mu \nu}=-\frac{i}{4} \operatorname{Tr} \sigma^{\mu \alpha \dot{\alpha}}\left\{\bar{Q}_{\dot{\alpha}}, J_{\alpha}^{\nu}\right\}
$$

where $J_{\alpha}^{\nu}$ is the susy Noether current.

The antisymmetric part of $T^{\mu \nu}$ gives the standard expression for the central charge density, while the symmetric part is a genuine momentum in the extra dimension:

$$
\langle Z\rangle=\int d^{2} x\left\langle T^{03}\right\rangle=\left\langle\tilde{Z}+\tilde{P}_{3}\right\rangle .
$$

(A similar decomposition is valid for the kink [39].)

$\tilde{Z}$ corresponds to the classical expression for the central charge. Being a surface term, its quantum corrections can be evaluated at infinity:

$$
\langle\tilde{Z}\rangle=\int d^{2} x \partial_{k} \epsilon_{k l}\left\langle\tilde{\zeta}_{l}\right\rangle=\left.\int_{0}^{2 \pi} d \theta\left\langle\tilde{\zeta}_{\theta}\right\rangle\right|_{r \rightarrow \infty}
$$

with $\tilde{\zeta}_{l}=e v_{0}^{2} A_{l}-i \phi^{\dagger} D_{l} \phi$ and $v_{0}^{2}=v^{2}+\delta v^{2}$.

Expanding in quantum fields $\phi=\phi_{\mathrm{V}}+\eta, A=A^{\mathrm{V}}+a$ and using that the classical fields $\phi_{\mathrm{V}} \rightarrow v e^{i n \theta}, A_{\theta}^{\mathrm{V}} \rightarrow n / e$, $D_{\theta}^{\mathrm{V}} \phi_{\mathrm{V}} \rightarrow 0$ as $r \rightarrow \infty$, we obtain to one-loop order

$$
\begin{aligned}
\langle\tilde{Z}\rangle= & 2 \pi n v_{0}^{2} \\
- & \left.i \int_{0}^{2 \pi} d \theta\left\langle\left(\phi_{\mathrm{V}}^{*}+\eta^{\dagger}\right)\left(D_{\theta}^{\mathrm{V}}-i e a_{\theta}\right)\left(\phi_{\mathrm{V}}+\eta\right)\right\rangle\right|_{r \rightarrow \infty} \\
= & 2 \pi n\left\{v_{0}^{2}-\left.\left\langle\eta^{\dagger} \eta\right\rangle\right|_{r \rightarrow \infty}\right\} \\
& -i \int_{0}^{2 \pi} d \theta\left\{\left\langle\eta^{\dagger} \partial_{\theta} \eta\right\rangle\right. \\
& \left.-i e \phi_{\mathrm{V}}^{*}\left\langle a_{\theta} \eta\right\rangle-i e \phi_{\mathrm{V}}\left\langle a_{\theta} \eta^{\dagger}\right\rangle\right\}\left.\right|_{r \rightarrow \infty} \\
\equiv & Z_{a}+Z_{b}
\end{aligned}
$$

where we have used $\langle\eta(r \rightarrow \infty)\rangle \rightarrow 0$ (which determines $\left.\delta v^{2}\right), \int_{0}^{2 \pi} d \theta\left\langle a_{\theta}\right\rangle=0$, and $\left\langle\eta^{\dagger} \eta a_{\theta}\right\rangle=O\left(\hbar^{2}\right)$.

The first contribution, $Z_{a}$, can be easily evaluated for arbitrary gauge parameter $\xi$, yielding

$$
\begin{aligned}
Z_{a} & =2 \pi n\left\{v_{0}^{2}-\left.\frac{1}{2}(\langle\sigma \sigma\rangle+\langle\rho \rho\rangle)\right|_{r \rightarrow \infty}\right\} \\
& =2 \pi n\left\{v_{0}^{2}-\frac{1}{2}\left[I(m)+I\left(\xi^{\frac{1}{2}} m\right)\right]\right\} \\
& =2 \pi n\left(v_{0}^{2}-\delta v^{2}\right)=2 \pi n v^{2} .
\end{aligned}
$$

If this was all, the quantum corrections to $Z$ would cancel, just as in the naive calculation of $Z$ in the susy kink [20,21].

The second contribution in (63), however, does not vanish when taking the limit $r \rightarrow \infty$. This contribution is simplest in the $\xi=1$ gauge, where the $\eta$ and $a_{\theta}$ fluctuations are governed by the fluctuation equations (48). In the limit $r \rightarrow \infty$ one has $\left|\phi_{\mathrm{V}}\right| \rightarrow v$ and $D_{-} \phi_{\mathrm{V}} \rightarrow 0$ exponentially. This eliminates the contributions from $\left\langle a_{\theta} \eta\right\rangle$. However, $D_{k}^{2}$, which governs the $\eta$ fluctuations, contains long-range contributions from the vector potential. Making a separation of variables in $r$ and $\theta$ one finds that asymptotically

$$
\left|D_{k}^{\mathrm{V}} \eta\right|^{2} \rightarrow\left|\partial_{r} \eta\right|^{2}+\frac{1}{r^{2}}\left|\left(\partial_{\theta}-i n\right) \eta\right|^{2}
$$

so that the $\eta$ fluctuations have an extra phase factor $e^{i n \theta}$ compared to those in the trivial vacuum. We thus have, in the $\xi=1$ gauge,

$$
\begin{aligned}
Z_{b} & =-i \int_{0}^{2 \pi} d \theta\left\{\left\langle\eta^{\dagger} \partial_{\theta} \eta\right\rangle\right. \\
& \left.\quad-i e \phi_{\mathrm{V}}^{*}\left\langle a_{\theta} \eta\right\rangle-i e \phi_{\mathrm{V}}\left\langle a_{\theta} \eta^{\dagger}\right\rangle\right\}\left.\right|_{r \rightarrow \infty} \\
= & -i \int_{0}^{2 \pi} d \theta\left\langle\eta^{\dagger} \partial_{\theta} \eta\right\rangle_{\xi=1}=2 \pi n\left\langle\eta^{\dagger} \eta\right\rangle_{\xi=1, r \rightarrow \infty} \\
= & \left.2 \pi n \delta v^{2}\right|_{\xi=1} .
\end{aligned}
$$

This is exactly the result for the one-loop correction to the mass of the vortex in eq. (59), implying saturation of the BPS bound provided that there are now no anomalous contributions to the central charge operator as there are in the case in the $N=1$ susy kink [39].

In dimensional regularization by dimensional reduction from a higher-dimensional model such anomalous contributions to the central charge operator come from a finite remainder of the extra momentum operator [39]. The latter is given by [55] 


$$
Z_{c}=\left\langle\tilde{P}_{3}\right\rangle=\int d^{2} x\left\langle F_{0 i} F_{3 i}+\left(D_{0} \phi\right)^{\dagger} D_{3} \phi+\left(D_{3} \phi\right)^{\dagger} D_{0} \phi-i \bar{\chi} \bar{\sigma}_{0} \partial_{3} \chi-i \bar{\psi} \bar{\sigma}_{0} D_{3} \psi\right\rangle .
$$

Inserting mode expansions for the quantum fields one immediately finds that the bosonic contributions vanish because of symmetry in the extra trivial dimension. However, this is not the case for the fermionic fields, which have a mode expansion of the form

$$
\left(\begin{array}{l}
U \\
V
\end{array}\right)=\int \frac{d^{\epsilon} \ell}{(2 \pi)^{\epsilon / 2}} \oiint_{k} \frac{1}{\sqrt{2 \omega}}\left\{b_{k, \ell} e^{-i(\omega t-\ell z)}\left(\begin{array}{c}
\sqrt{\omega-\ell} u_{1} \\
\sqrt{\omega-\ell} u_{2} \\
\sqrt{\omega+\ell} v_{1} \\
\sqrt{\omega+\ell} v_{2}
\end{array}\right)+d_{k, \ell}^{\dagger} \times(c . c .)\right\},
$$

where we have not written out explicitly the zero-modes (for which $\omega^{2}=\ell^{2}$ ). The fermionic contribution to $Z_{c}$ reads, schematically,

$$
\begin{aligned}
Z_{c} & =\left\langle\tilde{P}_{3}\right\rangle= \\
& \int \frac{d^{\epsilon} \ell}{(2 \pi)^{\epsilon}} \Sigma \int_{k} \frac{\ell^{2}}{2 \omega} \int d^{2} x\left[\left|u_{1}\right|^{2}+\left|u_{2}\right|^{2}-\left|v_{1}\right|^{2}-\left|v_{2}\right|^{2}\right]
\end{aligned}
$$

where $\omega=\sqrt{\omega_{k}+\ell^{2}}$, so that the $\ell$ integral does give a nonvanishing result. However, the $x$-integration over the mode functions $u_{1,2}(k ; x)$ and $v_{1,2}(k ; x)$ produces their spectral densities, which cancel up to zero-mode contributions as we have seen above ${ }^{11}$, and zero-mode contributions only produce scaleless integrals which vanish in dimensional regularization. Hence, $Z_{c}=0$ and $|Z|=\left|Z_{a}+Z_{b}\right|=E$, so that the BPS bound is saturated at the (one-loop) quantum level.

\section{The (susy) monopole.}

We now consider the $N=2$ monopole in $3+1$ dimensions, which has been used by many authors in studies of duality. The monopole model has more unbroken susy generators than the susy kink or the vortex, so one runs the risk (or the blessing) of vanishing quantum corrections. This model has been studied before in Refs. [62, 63, 64] and while the initial result of vanishing corrections of Ref. [62] turned out to be an oversimplification, Refs. [63, 64] nevertheless arrived at the conclusion of vanishing quantum corrections, at least in the simplest renormalization schemes. Using susy-preserving dimensional regularization by dimensional reduction, we have instead recently shown [65] that there are nonvanishing but equal quantum corrections to both mass and central charge of the $N=2$ monopole. Thus BPS saturation is preserved as required by multiplet shortening arguments [3], but in a nontrivial manner.

The $N=2$ super-Yang-Mills theory in $3+1$ dimensions can be obtained by dimensional reduction from the $(5+1)$ - dimensional $N=1$ theory [66]

$$
\mathcal{L}=-\frac{1}{4} F_{A B}^{2}-\bar{\lambda} \Gamma^{A} D_{A} \lambda,
$$

where the indices $A, B$ take the values $0,1,2,3,5,6$ and which is invariant under

$$
\delta A_{B}^{a}=\bar{\lambda}^{a} \Gamma_{B} \eta-\bar{\eta} \Gamma_{B} \lambda^{a}, \quad \delta \lambda^{a}=\frac{1}{2} F_{B C}^{a} \Gamma^{B} \Gamma^{C} \eta .
$$

The complex spinor $\lambda$ is in the adjoint representation of the gauge group which we assume to be $\mathrm{SU}(2)$ in the following and $\left(D_{A} \lambda\right)^{a}=\left(\partial_{A} \lambda+g A_{A} \times \lambda\right)^{a}$ $=\partial_{A} \lambda^{a}+g \epsilon^{a b c} A_{A}^{b} \lambda^{c}$. Furthermore, $\lambda$ and $\eta$ satisfy the Weyl condition:

$$
\left(1-\Gamma_{7}\right) \lambda=0 \quad \text { with } \quad \Gamma_{7}=\Gamma_{0} \Gamma_{1} \Gamma_{2} \Gamma_{3} \Gamma_{5} \Gamma_{6} .
$$

To carry out the dimensional reduction we write $A_{B}=$ $\left(A_{\mu}, P, S\right)$ and choose the following representation of gamma matrices

$$
\begin{array}{ccl}
\Gamma_{\mu}=\gamma_{\mu} \otimes \sigma_{1} & , \quad \mu=0,1,2,3, \\
\Gamma_{5}=\gamma_{5} \otimes \sigma_{1} & , \quad \Gamma_{6}=\mathbb{1} \otimes \sigma_{2} .
\end{array}
$$

In this representation the Weyl condition (72) becomes $\lambda=$ $\left(\begin{array}{c}\psi \\ 0\end{array}\right)$, with a complex four-component spinor $\psi{ }^{12}$

The (3+1)-dimensional Lagrangian then reads

$$
\begin{aligned}
& \mathcal{L}=-\left\{\frac{1}{4} F_{\mu \nu}^{2}+\frac{1}{2}\left(D_{\mu} S\right)^{2}+\frac{1}{2}\left(D_{\mu} P\right)^{2}+\frac{1}{2} g^{2}(S \times P)^{2}\right\} \\
& -\left\{\bar{\psi} \gamma^{\mu} D_{\mu} \psi+i g \bar{\psi}(S \times \psi)+g \bar{\psi} \gamma_{5}(P \times \psi)\right\} .
\end{aligned}
$$

We choose the symmetry-breaking Higgs field as $S^{a} \equiv$ $A_{6}^{a}=v \delta_{3}^{a}$ in the trivial sector. The BPS monopoles are of the form (for $A_{0}=0$ ) [67]

$$
\begin{aligned}
A_{i}^{a} & =\epsilon_{a i j} \frac{x^{j}}{g r^{2}}(1-K(m r)), \\
S^{a} & =\delta_{i}^{a} \frac{x^{i}}{g r^{2}} H(m r),
\end{aligned}
$$

with $H=m r \operatorname{coth}(m r)-1$ and $K=m r / \sinh (m r)$, where $m=g v$ is the mass of the particles that are charged under the unbroken U(1). The BPS equation $F_{i j}^{a}+$

\footnotetext{
${ }^{11}$ An explicit calculation which confirms these cancellations can be found in [16].

${ }^{12}$ We use the metric with signature $(-,+,+,+,+,+)$ and $\bar{\lambda}^{a}=\left(\lambda^{a}\right)^{\dagger} i \Gamma^{0}$, hence $\bar{\psi}^{a}=\left(\psi^{a}\right)^{\dagger} i \gamma^{0}$. One can rewrite this model in terms of two symplectic Majorana spinors in order to exhibit the $R$ symmetry group $\mathrm{U}(2)$.
} 
$\epsilon_{i j k} D_{k} S^{a}=0$ can be written as a self-duality equation for $F_{M N}$ with $M, N=1,2,3,6$, and the classical mass is $M_{\mathrm{cl} .}=4 \pi m / g^{2}$.

The susy algebra for the charges $Q^{\alpha}=\int j^{0 \alpha} d^{3} x$ with $j^{A}=\frac{1}{2} \Gamma^{B} \Gamma^{C} F_{B C} \Gamma^{A} \lambda$ reads

$$
\left\{Q^{\alpha}, \bar{Q}_{\beta}\right\}=-\left(\gamma^{\mu}\right)^{\alpha}{ }_{\beta} P_{\mu}+\left(\gamma_{5}\right)^{\alpha}{ }_{\beta} U+i \delta_{\beta}^{\alpha} V
$$

with $\alpha, \beta=1, \ldots, 4$. In the trivial sector $P_{\mu}$ acts as $\partial_{\mu}$, and $U$ multiplies the massive fields by $m$, but in the topological sector $P_{\mu}$ are covariant translations, and $U$ and $V$ are surface integrals. The classical monopole solution saturates the BPS bound $M^{2} \geq|\langle U\rangle|^{2}+|\langle V\rangle|^{2}$ by $\left|U_{\text {cl. }}\right|=M_{\text {cl. }}$, and $V_{\text {cl. }}=0$.

For obtaining the one-loop quantum corrections, one has to consider quantum fluctuations about the monopole background. The bosonic fluctuation equations turn out to be simplest in the background-covariant Feynman- $R_{\xi}$ gauge which is obtained by dimensional reduction of the ordinary background-covariant Feynman gauge-fixing term in $(5+1)$ dimensions $-\frac{1}{2}\left(D_{B}[\hat{A}] a^{B}\right)^{2}$, where $a^{B}$ comprises the bosonic fluctuations and $\hat{A}^{B}$ the background fields. As has been found in Refs. [63, 64], in this gauge the eigenvalues of the bosonic fluctuation equations (taking into account Faddeev-Popov fields) and those of the fermionic fluctuation equations combine such that one can make use of an indextheorem by Weinberg [68] to determine the spectral density. This leads to the following (unregularized!) formula for the one-loop mass correction

$$
\begin{aligned}
M^{(1)} & =\frac{4 \pi m_{0}}{g_{0}^{2}}+\frac{\hbar}{2} \sum\left(\omega_{B}-\omega_{F}\right) \\
& =\frac{4 \pi m_{0}}{g_{0}^{2}}+\frac{\hbar}{2} \int \frac{d^{3} k}{(2 \pi)^{3}} \sqrt{k^{2}+m^{2}} \rho_{M}\left(k^{2}\right),
\end{aligned}
$$

with $m_{0}$ and $g_{0}$ denoting bare quantities and

$$
\rho_{M}\left(k^{2}\right)=\frac{-8 \pi m}{k^{2}\left(k^{2}+m^{2}\right)} .
$$

This expression is logarithmically divergent and is made finite by combining it with the one-loop renormalization of $g$, while $m$ does not need to be renormalized [63, 64]. Combining these two expressions from the sum over zero point energies and the counter term, we find that there is a mismatch proportional to $\epsilon$, but $\epsilon$ multiplies a logarithmically divergent integral, which in dimensional regularization involves a pole $\epsilon^{-1}$. We therefore obtain a finite correction of the form

$$
\begin{aligned}
M^{(1)}=\frac{4 \pi m}{g^{2}}-\epsilon & \times \frac{2 m}{\pi} \frac{\Gamma\left(-\frac{1}{2}-\frac{\epsilon}{2}\right)}{\left(2 \pi^{\frac{1}{2}}\right) \epsilon\left(-\frac{1}{2}\right)} \\
\times \int_{0}^{\infty} d k\left(k^{2}+m^{2}\right)^{-\frac{1}{2}+\frac{\epsilon}{2}} & \\
= & \frac{4 \pi m}{g^{2}}-\frac{2 m}{\pi}+O(\epsilon)
\end{aligned}
$$

which because of the fact that it arises as $0 \times \infty$ bears the hallmark of an anomaly.

Indeed, as we shall now show, this result is completely analogous to the case of the $N=1$ susy kink in
$(1+1)$ dimensions, where a nonvanishing quantum correction to the kink mass (in a minimal renormalization scheme) is associated with an anomaly in the central charge (which is scheme-independent; in a non-minimal renormalization scheme there are also non-anomalous quantum corrections to the central charge).

In Ref. [64] it has been argued that in the renormalization scheme defined above, the one-loop contributions to the central charge precisely cancel the contribution from the counterterm in the classical expression. In this particular calculation it turns out that the cancelling contributions have identical form so that the regularization methods of Ref. [64] can be used at least self-consistently, and also straightforward dimensional regularization would imply complete cancellations. The result (80) would then appear to violate the Bogomolnyi bound.

However, this is just the situation encountered in the (1+1)-dimensional susy kink. As we have shown in Ref. [39] and recapitulated above, dimensional regularization gives a zero result for the correction to the central charge unless the latter is augmented by the momentum operator in the extra dimension used to embed the soliton. This is necessary for manifest supersymmetry, and, indeed, the extra momentum operator can acquire a nonvanishing expectation value. As it turns out, the latter is entirely due to nontrivial contributions from the fermions $\psi=\left(\begin{array}{c}\psi_{+} \\ \psi_{-}\end{array}\right)$, whose fluctuation equations have the form

$$
\begin{aligned}
L \psi_{+}+i\left(\partial_{t}+\partial_{5}\right) \psi_{-} & =0 \\
i\left(\partial_{t}-\partial_{5}\right) \psi_{+}+L^{\dagger} \psi_{-} & =0 .
\end{aligned}
$$

The fermionic field operator can be written as

$$
\begin{aligned}
& \psi(x)= \int \frac{d^{\epsilon} \ell}{(2 \pi)^{\epsilon / 2}} \sum \frac{d^{3} k}{(2 \pi)^{3 / 2}} \frac{1}{\sqrt{2 \omega}} \\
& \times\left\{a_{k l} e^{-i\left(\omega t-\ell x^{5}\right)}\left(\begin{array}{c}
\sqrt{\omega-\ell} \chi_{+} \\
-\sqrt{\omega+\ell} \chi_{-}
\end{array}\right)\right. \\
&\left.+b_{k l}^{\dagger} e^{i\left(\omega t-\ell x^{5}\right)}\left(\begin{array}{l}
\sqrt{\omega-\ell} \chi_{+} \\
\sqrt{\omega+\ell} \chi_{-}
\end{array}\right)\right\}
\end{aligned}
$$

where $\chi_{-}=\frac{1}{\omega_{k}} L \chi_{+}$and $\chi_{+}=\frac{1}{\omega_{k}} L^{\dagger} \chi_{-}$with $\omega_{k}^{2}=$ $k^{2}+m^{2}$, and the normalization factors $\sqrt{\omega \pm \ell}$ are such that $L^{\dagger} L \chi_{+}=\omega^{2} \chi_{+}$and $L L^{\dagger} \chi_{-}=\omega^{2} \chi_{-}$with $\omega^{2}=\omega_{k}^{2}+\ell^{2}$. Because of these normalization factors, one obtains an expression for the momentum density $\Theta_{05}$ in the extra dimension which has an even component under reflection in the extra momentum variable $\ell$

$$
\begin{array}{r}
\left\langle\Theta_{05}\right\rangle=\int \frac{d^{\epsilon} \ell}{(2 \pi)^{\epsilon}} \int \frac{d^{3} k}{(2 \pi)^{3}} \frac{\ell}{2 \omega}\left[(\omega-\ell)\left|\chi_{+}\right|^{2}\right. \\
\left.+(\omega+\ell)\left|\chi_{-}\right|^{2}\right] \\
=\int \frac{d^{\epsilon} \ell}{(2 \pi)^{\epsilon}} \int \frac{d^{3} k}{(2 \pi)^{3}} \frac{\ell^{2}}{2 \omega}\left(\left|\chi_{-}\right|^{2}-\left|\chi_{+}\right|^{2}\right)
\end{array}
$$

(omitting zero-mode contributions which do not contribute in dimensional regularization [39]).

Integration over $x$ then produces the spectral density (79) and finally yields 


$$
\begin{aligned}
\Delta U_{\text {an }} & =\int d^{3} x\left\langle\Theta_{05}\right\rangle \\
& =\int \frac{d^{3} k d^{\epsilon} \ell}{(2 \pi)^{3+\epsilon}} \frac{\ell^{2}}{2 \sqrt{k^{2}+\ell^{2}+m^{2}}} \rho_{M}\left(k^{2}\right) \\
& =-4 m \int_{0}^{\infty} \frac{d k}{2 \pi} \int \frac{d^{\epsilon} \ell}{(2 \pi)^{\epsilon}} \frac{\ell^{2}}{\left(k^{2}+m^{2}\right) \sqrt{k^{2}+\ell^{2}+m^{2}}} \\
& =-8 \frac{\Gamma\left(1-\frac{\epsilon}{2}\right)}{(4 \pi)^{1+\frac{\epsilon}{2}}} \frac{m^{1+\epsilon}}{1+\epsilon}=-\frac{2 m}{\pi}+O(\epsilon),
\end{aligned}
$$

which is indeed equal to the nonzero mass correction obtained above.

This verifies that the BPS bound remains saturated under quantum corrections, but the quantum corrections to mass and central charge both contain an anomalous contribution, analogous to the central-charge anomaly in the $1+1$ dimensional minimally supersymmetric kink.

The nontrivial result (85) is in fact in complete accordance with the low-energy effective action for $N=2$ superYang-Mills theory as obtained by Seiberg and Witten [2]. ${ }^{13}$ According to the latter, the low-energy effective action is fully determined by a prepotential $\mathcal{F}(A)$, which to one-loop order is given by

$$
\mathcal{F}_{1-\text { loop }}(A)=\frac{i}{2 \pi} A^{2} \ln \frac{A^{2}}{\Lambda^{2}},
$$

where $A$ is a chiral superfield and $\Lambda$ the scale parameter of the theory generated by dimensional transmutation. The value of its scalar component $a$ corresponds in our notation to $g v=m$. In the absence of a $\theta$ parameter, the one-loop renormalized coupling is given by

$$
\frac{4 \pi i}{g^{2}}=\tau(a)=\frac{\partial^{2} \mathcal{F}}{\partial a^{2}}=\frac{i}{\pi}\left(\ln \frac{a^{2}}{\Lambda^{2}}+3\right) .
$$

This definition agrees with the "minimal" renormalization scheme that we have considered above, because the latter involves only the zero-momentum limit of the two-point function of the massless fields. For a single magnetic monopole, the central charge is given by

$$
|U|=a_{D}=\frac{\partial \mathcal{F}}{\partial a}=\frac{i}{\pi} a\left(\ln \frac{a^{2}}{\Lambda^{2}}+1\right)=\frac{4 \pi a}{g^{2}}-\frac{2 a}{\pi},
$$

and since $a=m$, this exactly agrees with the result of our direct calculation in (85).

Now, the low-energy effective action associated with (86) has been derived from a consistency requirement with the anomaly of the $\mathrm{U}(1)_{R}$ symmetry of the microscopic theory. The central-charge anomaly, which we have identified as being responsible for the entire nonzero correction (85), is evidently consistent with the former. Just as in the case of the minimally supersymmetric kink in $1+1$ dimensions, it constitutes a new anomaly ${ }^{14}$ that had previously been ignored in direct calculations $[63,64]$ of the quantum corrections to the $N=2$ monopole.

\section{References}

[1] R. Rajaraman, Solitons and Instantons (North-Holland, Amsterdam, 1982).

[2] N. Seiberg and E. Witten, Nucl. Phys. B426, 19 (1994); Nucl. Phys. B431, 484 (1994); L. Alvarez-Gaume, S. F. Hassan, Fortsch. Phys. 45, 159 (1997).

[3] E. Witten and D. Olive, Phys. Lett. B78, 97 (1978).

[4] G. 't Hooft and M. Veltman, Nucl. Phys. B44, 189 (1972).

[5] C. G. Bollini, J. J. Giambiagi, CBPF-NF-085/83; E. Brézin, S. Feng, Phys. Rev. B29, 472 (1984); G. Münster, Nucl. Phys. B324, 630 (1989); A. Parnachev, L. G. Yaffe, Phys. Rev. D62, 105034 (2000).

[6] W. Siegel, Phys. Lett. B84 (1979) 193; Phys. Lett. B94 (1980) 37; D. M. Capper, D. R. T. Jones, P. van Nieuwenhuizen, Nucl. Phys. B167 (1980) 479.

[7] C. G. Callan, J. A. Harvey, Nucl. Phys. B250, 427 (1985); G. W. Gibbons, N. D. Lambert, Phys. Lett. B488, 90 (2000).

[8] L. Da Rold, C. D. Fosco, and A. López, J. Math. Phys. 44, 588 (2003).

[9] C. Aragão de Carvalho, G. C. Marques, A. J. da Silva, and I. Ventura, Nucl. Phys. B265, 45 (1986).

[10] N. Graham, R. L. Jaffe, M. Quandt, and H. Weigel, Phys. Rev. Lett. 87, 131601 (2001).

[11] C. A. A. de Carvalho, Phys. Rev. D65, 065021 (2002), E: Phys. Rev. D66, 049901 (2002); A. Bessa, C. A. A. de Carvalho, and E. S. Fraga, hep-ph/0402091.

[12] A. A. Izquierdo, et al., hep-th/0311057.

[13] P. Horava and E. Witten, Nucl. Phys. B475, 94 (1996).

[14] G. Bonneau, Nucl. Phys. B167, 261 (1980) 261.

[15] A. S. Goldhaber, A. Rebhan, P. van Nieuwenhuizen, and R. Wimmer, hep-th/0211087.

\footnotetext{
${ }^{13} \mathrm{We}$ are grateful to Horatiu Nastase for pointing this out to us.

${ }^{14}$ The possibility of central-charge anomalies in $N=2$ super-Yang-Mills theories has most recently also been noted in [69], however without a calculation of the coefficients.
} 
[16] A. S. Goldhaber, A. Rebhan, P. van Nieuwenhuizen, and R. Wimmer, Phys. Rept. 398, 179 (2004).

[17] A. D’Adda, P. Di Vecchia, Phys. Lett. B73, 162 (1978); A. D'Adda, R. Horsley, and P. Di Vecchia, Phys. Lett. B76, 298 (1978); R. Horsley, Nucl. Phys. B151, 399 (1979).

[18] J. F. Schonfeld, Nucl. Phys. B161, 125 (1979).

[19] R. K. Kaul and R. Rajaraman, Phys. Lett. B131, 357 (1983); A. Chatterjee, P. Majumdar, Phys. Rev. D30, 844 (1984); Phys. Lett. B159, 37 (1985); H. Yamagishi, Phys. Lett. B147, 425 (1984)

[20] C. Imbimbo and S. Mukhi, Nucl. Phys. B247, 471 (1984).

[21] A. Rebhan and P. van Nieuwenhuizen, Nucl. Phys. B508, 449 (1997).

[22] R. F. Dashen, B. Hasslacher, and A. Neveu, Phys. Rev. D11, 3424 (1975).

[23] A. Uchiyama, Prog. Theor. Phys. 75, 1214 (1986).

[24] H. Nastase, M. Stephanov, P. van Nieuwenhuizen, and A. Rebhan, Nucl. Phys. B542, 471 (1999).

[25] A. S. Goldhaber, A. Litvintsev, and P. van Nieuwenhuizen, Phys. Rev. D64, 045013 (2001).

[26] A. S. Goldhaber, A. Rebhan, P. van Nieuwenhuizen, and R. Wimmer, Phys. Rev. D66, 085010 (2002).

[27] M. A. Shifman, A. I. Vainshtein, and M. B. Voloshin, Phys. Rev. D59, 045016 (1999).

[28] A. Losev, M. A. Shifman, and A. I. Vainshtein, Phys. Lett. B522, 327 (2001); New J. Phys. 4, 21 (2002).

[29] N. Graham and R. L. Jaffe, Nucl. Phys. B544, 432 (1999).

[30] A. Litvintsev and P. van Nieuwenhuizen, Once more on the BPS bound for the SUSY kink, hep-th/0010051.

[31] A. S. Goldhaber, A. Litvintsev, and P. van Nieuwenhuizen, Phys. Rev. D67, 105021 (2003).

[32] R. Wimmer, Quantization of supersymmetric solitons, Master thesis, hep-th/0109119, 2001.

[33] M. Bordag, A. S. Goldhaber, P. van Nieuwenhuizen, and D. Vassilevich, Phys. Rev. D66, 125014 (2002).

[34] J. Casahorrán, J. Phys. A22, L413 (1989).

[35] L. J. Boya and J. Casahorrán, J. Phys. A23, 1645 (1990).

[36] K. Cahill, A. Comtet, and R. J. Glauber, Phys. Lett. B64, 283 (1976).

[37] J. Casahorrán, J. Phys. A22, L1167 (1989).

[38] A. Rebhan, P. van Nieuwenhuizen, and R. Wimmer, New J. Phys. 4, 31 (2002).

[39] A. Rebhan, P. van Nieuwenhuizen, and R. Wimmer, Nucl. Phys. B648, 174 (2003).

[40] P. Di Vecchia and S. Ferrara, Nucl. Phys. B130, 93 (1977).

[41] G. W. Gibbons and P. K. Townsend, Phys. Rev. Lett. 83, 1727 (1999).
[42] E. Witten, Nucl. Phys. B202, 253 (1982).

[43] F. Cooper, A. Khare, and U. Sukhatme, Supersymmetry in Quantum Mechanics (World Sci., Singapore, 2001).

[44] K. Fujikawa and P. van Nieuwenhuizen, Ann. Phys. 308, 78 (2003); K. Fujikawa, A. Rebhan, and P. van Nieuwenhuizen, Int. J. Mod. Phys. A18, 5637 (2003).

[45] A. Rebhan, P. van Nieuwenhuizen, and R. Wimmer, Nucl. Phys. B679, 382 (2004).

[46] A. A. Abrikosov, Sov. Phys. JETP 5, 1174 (1957).

[47] H. B. Nielsen and P. Olesen, Nucl. Phys. B61, 45 (1973).

[48] H. J. de Vega and F. A. Schaposnik, Phys. Rev. D14, 1100 (1976).

[49] C. H. Taubes, Commun. Math. Phys. 72, 277 (1980).

[50] J. R. Schmidt, Phys. Rev. D46, 1839 (1992).

[51] J. Edelstein, C. Núñez, and F. Schaposnik, Phys. Lett. B329, 39 (1994).

[52] A. Gorsky and M. A. Shifman, Phys. Rev. D61, 085001 (2000).

[53] A. I. Vainshtein and A. Yung, Nucl. Phys. B614, 3 (2001).

[54] E. B. Bogomolnyi, Sov. J. Nucl. Phys. 24, 449 (1976).

[55] B.-H. Lee and H. Min, Phys. Rev. D51, 4458 (1995).

[56] D. V. Vassilevich, Phys. Rev. D68, 045005 (2003).

[57] R. Wimmer, PhD thesis, Techn. Univ. Vienna, 2003.

[58] B.-H. Lee, C.-k. Lee, and H. Min, Phys. Rev. D45, 4588 (1992).

[59] E. J. Weinberg, Phys. Rev. D24, 2669 (1981).

[60] K. Hori and C. Vafa, Mirror symmetry, hep-th/0002222.

[61] G. 't Hooft, Nucl. Phys. B35, 167 (1971).

[62] A. D’Adda, R. Horsley, and P. Di Vecchia, Phys. Lett. B76, 298 (1978).

[63] R. K. Kaul, Phys. Lett. B143, 427 (1984)

[64] C. Imbimbo and S. Mukhi, Nucl. Phys. B249, 143 (1985).

[65] A. Rebhan, P. van Nieuwenhuizen, and R. Wimmer, Phys. Lett. B 594, 234 (2004).

[66] L. Brink, J. H. Schwarz, and J. Scherk, Nucl. Phys. B121, 77 (1977).

[67] M. K. Prasad and C. M. Sommerfield, Phys. Rev. Lett. 35, 760 (1975)

[68] E. J. Weinberg, Phys. Rev. D20, 936 (1979).

[69] M. Shifman and A. Yung, hep-th/0312257.

[70] R. Jackiw, Ann. Phys. 129, 183 (1980); Ann. Phys. 201, 83 (1990); B. R. Holstein, Am. J. Phys. 61, 142 (1993). 